\title{
SYMMETRY AND VARIATION OF HODGE STRUCTURES *
}

\author{
I. C. BAUER ${ }^{\dagger}$ AND F. CATANESE ${ }^{\dagger}$
}

This article is dedicated to Yum Tong Siu's 60-th birthday

0. Introduction. The Torelli theorem (cf.[Tor13], [Andr58], [Weil-57]) states that two algebraic curves are isomorphic if and only if their Jacobian varieties are isomorphic as polarized abelian varieties.

André Weil ([Weil-CPII]) set up a program for doing arithmetics on K3 surfaces, based on a Torelli type theorem, which was later proven through the effort of several authors (for this long story and related references we refer to Chapter VIII of the book [BPV]). This result is crucial for answering questions about the existence of K3 surfaces or families thereof possessing certain curve configurations.

The general Torelli question, as set up by Griffiths ([Grif68], [Grif70], cf. also [Grif-Schmid], [Grif84]), is to associate to each projective variety $X$ of general type its Hodge structure of weight $n=\operatorname{dim}(X)$, and ask whether the corresponding "period map" $\psi_{n}$ is injective on the local moduli space (or Kuranishi space of $X$ ).

It was known since long time that, as soon as the dimension is at least two, there are families of varieties without Hodge structures, which are not rigid. Surfaces of general type with $q=p_{g}=0$ were constructed in the 30's by Campedelli and Godeaux ([Cam32], [God35]), and for instance, in the case of the Godeaux surfaces, the Kuranishi family has dimension 8.

A natural question which arises is: under which hypothesis on $X$ is a local Torelli theorem valid for the Hodge structure of weight $n=\operatorname{dim} X$ ?

In other words, when is the local period map $\psi_{n}$ a local embedding?

The question is already quite open in dimension $n=2$, and the hypothesis of requiring $X$ to be simply connected only made the search of counterexamples more complicated (cf. e.g. [Cat84] for a brief account of these examples).

On the other hand, Lieberman, Peters and Wilsker (cf. [L-P-W77]) made clear that, thanks to Griffiths' interpretation of the derivative of the period map as a cupproduct (cf. further below in the introduction), the infinitesimal Torelli theorem (injectivity of the derivative) would follow from the vanishing of a certain Koszul cohomology group.

This approach was later developed by several authors (cf. e.g. [Gren84], [Gren85], [Flen86], [Cox87]) who essentially proved that, given a construction involving some degree $d$ (taking hypersurfaces of degree $d$ in some fixed manifold, or complete intersections), then infinitesimal Torelli holds for $d$ sufficiently large. In some sense, results of this kind parallel Serre's vanishing theorem $B(n)$, and it would be beautiful to give precise geometrical conditions which would ensure the validity of the infinitesimal Torelli theorem, e.g. for varieties of general type with ample canonical bundle.

Certainly it has been up to now an open question whether the condition that the canonical system be very ample is sufficient for this purpose (the condition was not holding for all hitherto known counterexamples). Note that, if the canonical bundle is

\footnotetext{
*Received August 12, 2003; accepted for publication October 29, 2003.

${ }^{\dagger}$ Mathematisches Institut der Universität Bayreuth, Universitätsstr. 30, D-95447 Bayreuth, Germany (Ingrid.Bauer@uni-bayreuth.de; Fabrizio.Catanese@uni-bayreuth.de).
} 
ample but not very ample, the infinitesimal Torelli theorem already fails in dimension 1 , as the case of hyperelliptic curves already shows.

Unfortunately, we show in this paper that this condition might not be sufficient, indeed we provide a series of examples of surfaces of general type for which the infinitesimal Torelli theorem fails on the whole moduli space in the worst possible way, namely, the period map has all fibres of positive dimension. This holds in spite of the fact that the canonical system is generally quasi very ample (i.e., we prove that it is a birational morphism which yields a local embedding on the complement of a finite set).

Our examples do not rule out the possibility that for very ample canonical system the period map may be generically finite. Indeed, in our examples one recovers the "missing" Hodge structure from the geometry of the singular locus of the canonical image.

We raise therefore the question: what geometric properties are required, for a variety with very ample canonical system, for the validity of the infinitesimal Torelli theorem? For instance, in terms of the geometry of the canonical image?

We proceed now to a more detailed information, introducing the standard notation and our situation.

Let $X$ be a smooth algebraic variety over the complex numbers, which for simplicity we assume to have ample canonical bundle $K_{X}$. Then $K_{X}$ defines a natural polarization on $X$ and we know from the Kodaira - Spencer - Kuranishi theory (cf. $[\mathrm{K}-\mathrm{M}],[\mathrm{Kur} 65])$ that there exists a semiuniversal deformation $p: \mathcal{X} \longrightarrow\left(Y, y_{0}\right)$ of $X$. In particular, the tangent space $T_{Y, y_{0}}$ of $Y$ in $y_{0}$ is naturally isomorphic to $H^{1}\left(X, T_{X}\right)$ and the dimension of $Y$ in $y_{0}$ is at least $\operatorname{dim} H^{1}\left(X, T_{X}\right)-\operatorname{dim} H^{2}\left(X, T_{X}\right)$.

For each $k \in\{1, \ldots, \operatorname{dim} X\}$ we have a corresponding variation of Hodge structure

$$
\left(H_{\mathbb{Z}}=\mathcal{R}^{k} p_{*}(\mathbb{Z}), H^{p, q}(y), Q\right)
$$

where $p+q=k, H_{\mathbb{Z}} \otimes \mathbb{C}=\bigoplus_{p+q=k} H^{p, q}(y)$, and the polarization $Q$ is a quadratic form on $H_{\mathbb{Z}}$ for which the subspaces $H^{p, q}(y)$ are pairwise orthogonal. To this variation of Hodge structure there is associated a holomorphic map $\Phi: Y \longrightarrow D$, where $D$ is the classifying domain of polarized Hodge structures of type $\left(h^{k, 0}, h^{k-1,1}, \ldots, h^{0, k}\right)$ and $h^{p, q}=\operatorname{dim} H^{p, q}(y)$ (cf. e.g. [Grif-Schmid]).

The infinitesimal Torelli theorem is said to hold for $X$ if and only if the differential $d \Phi$ of $\Phi$ is injective on $T_{Y, y_{0}}$.

In the late sixties P. Griffiths ([Grif68], [Grif70]) showed that the differential $d \Phi$ of the period map is given by the map below, induced by cup product

$$
d \Phi: H^{1}\left(X, T_{X}\right) \longrightarrow \bigoplus_{p+q=k} \operatorname{Hom}\left(H^{p, q}(X), H^{p-1, q+1}(X)\right) .
$$

Classically, for a smooth curve $C$ the infinitesimal Torelli theorem holds iff $g(C)=1,2$ or iff $g(C) \geq 3$ and $C$ is not hyperelliptic.

P. Griffiths posed the problem to determine the class of varieties with $K_{X}$ (sufficiently) ample for which infinitesimal Torelli holds. Even if there are several counterexamples to the infinitesimal Torelli theorem known, there is still the hope 
that for projective manifolds with sufficiently ample canonical bundle the result should be true.

The simple underlying idea of this paper is the following: assume that $X$ is the quotient of a smooth variety $Z$ by the free action of a finite group $G$. Then, in the Griffiths cup product map, if we replace $Z$ by $X$, we must replace each cohomology group by the subspace of $G$-invariants.

However, even if infinitesimally Torelli holds for $Z$, it does not need to hold for $X$ any longer, by the simple algebraic observation that the tensor product of invariants is much smaller than the subspace of invariants in the tensor product of $G$-representations.

A similar philosophy was used by S. Usui ([Usui81]) to justify failure of infinitesimal Torelli for varieties $Z$ with automorphisms, and in [Cat89] to produce generalized examples of everywhere non reduced moduli spaces (in the latter paper one needed the action not to be free, whereas in the former one had no restriction, but the "bad" varieties $Z$ with special automorphisms were not generic in the moduli space).

So, we shall study here the situation for quotients of projective manifolds by a free action of a finite group and it will turn out that there are examples of surfaces having quasi very ample canonical bundle and for which infinitesimal Torelli fails.

The most natural candidates, however, do not suffice for our purposes: quotients of a hypersurface $Z \subset \mathbb{P}^{3}$ of degree $d$ by the action of a finite group $G$ of order $m$. The first example is given by the classical Godeaux surfaces $(d=m=5)$, for which however there is no Hodge structure, and the second simplest example, with $d=m=7$, satisfies already local Torelli at the general point (it does so at the quotient of the Fermat surface).

For this reason we have to resort to quotients of products of curves, the so called surfaces isogenous to a (higher) product, whose moduli spaces were thoroughly investigated in [Cat00] (cf. also [Cat03]).

The simplest examples will be the ones where the group $G$ is cyclic. When $G$ has order two, we will get positive dimensional fibres of the period map which are relatives of the positive dimensional fibres of the Prym map (cf. [Nara96]). This case however is not fully satisfactory because we want $K_{X}$ to be quasi very ample.

The following will be one of our main results.

THEOREM 0.1. For any natural number $k \geq 2$ there exists a family of surfaces $\mathcal{S}_{k}$ of dimension $3 k+2$ such that the following hold:

1) for each $S_{k} \in \mathcal{S}_{k}$ the infinitesimal Torelli map

$$
d \Phi_{2}: H^{1}\left(S_{k}, T_{S_{k}}\right) \longrightarrow \operatorname{Hom}\left(H^{2,0}\left(S_{k}\right), H^{1,1}\left(S_{k}\right)\right)
$$

has a kernel of dimension at least two, i.e., the period map $\Phi_{2}$ belonging to the Hodge structures of weight 2 has fibres of dimension at least two over each point.

2) for a general $S_{k} \in \mathcal{S}_{k}$ the canonical divisor $K_{S_{k}}$ is quasi very ample, i.e., it gives a birational morphism which yields a local embedding on the complement of a finite set; 3) $h^{1}\left(S_{k}, T_{S_{k}}\right)=3 k+2$; in particular $\mathcal{S}_{k}$ is a generically smooth irreducible connected component in the moduli space.

We emphasise once more here the important fact that the surfaces we are considering all have unobstructed local moduli spaces (i.e., the basis of the Kuranishi family is smooth, and indeed an open set in $H^{1}\left(S, T_{S}\right)$ ). 
After preliminaries in section 1, the above main theorem will be shown in sections 2 and 3 .

In section 4, however, we just take the opposite point of view and ask the converse question: since the Torelli theorem holds for curves, may it be that one can completely characterize the case where a global $n$-tuple Torelli theorem does not hold for varieties isogenous to a product of curves?

Here, $n$-tuple Torelli means that we can reconstruct the variety from all of its Hodge structure (i.e., we do not restrict only to weight $n=\operatorname{dim}(X))$.

The question looks interesting in general, but rather complicated already for surfaces. For this reason we content ourself with giving sufficient conditions for the validity of double Torelli for surfaces isogenous to a product (theorem 4.4). In proving this result, we establish some intermediate technical results which may be of independent interest (lemma 4.6., proposition 4.5.). These results concern the eigenspaces for the action of a cyclic group on an algebraic curve $C$ inside the space of holomorphic differentials on $C$.

Finally, in section 5, we discuss another series of examples where we are not able to show global double Torelli, but where the local period map is injective.

1. Symmetry imposes failure of Torelli type theorems. As already mentioned, in this paper we exploit the above main idea as a simple tool for providing generic counterexamples to Torelli theorems. A similar observation was made (in a different direction, however, cf. [Usui81]) to show that often the failure of Torelli theorems (especially infinitesimal Torelli) is due to the presence of symmetry on the variety under consideration.

Our situation is more specific: we assume that $G$ is a finite group acting freely on a smooth algebraic variety $Z$ of dimension $n$. Let $X$ be the quotient $Z / G$.

Then the infinitesimal Torelli theorem for the periods of holomorphic $n$-forms holds for $Z$ if and only if we have surjectivity of

$$
H^{n-1}\left(Z, \Omega_{Z}^{1}\right) \otimes H^{0}\left(Z, \Omega_{Z}^{n}\right) \longrightarrow H^{n-1}\left(Z, \Omega_{Z}^{1} \otimes \Omega_{Z}^{n}\right) .
$$

We remark that for $n=2$ infinitesimal Torelli for the periods of 2 -forms holds if and only if infinitesimal Torelli for weight 2 Hodge structures holds, i.e.

$$
d \Phi_{2}: H^{1}\left(Z, T_{Z}\right) \longrightarrow \bigoplus_{p+q=2} \operatorname{Hom}\left(H^{p, q}(Z), H^{p-1, q+1}(Z)\right)
$$

is injective.

Whence, if infinitesimal Torelli for the periods of $n$-forms holds for $Z$, we have surjectivity also of the map

$$
\left(H^{n-1}\left(Z, \Omega_{Z}^{1}\right) \otimes H^{0}\left(Z, \Omega_{Z}^{n}\right)\right)^{G} \rightarrow\left(H^{n-1}\left(Z, \Omega_{Z}^{1} \otimes \Omega_{Z}^{n}\right)\right)^{G}=H^{n-1}\left(X, \Omega_{X}^{1} \otimes \Omega_{X}^{n}\right) .
$$

For simplicity of notation assume that $G$ is abelian (a quite similar fact holds in the general case, we shall however concentrate ourselves on the more tractable case where $G$ is abelian): by Schur's lemma we have

$$
\left(H^{n-1}\left(Z, \Omega_{Z}^{1}\right) \otimes H^{0}\left(Z, \Omega_{Z}^{n}\right)\right)^{G}=\bigoplus_{\chi \in G^{*}} H^{n-1}\left(Z, \Omega_{Z}^{1}\right)^{\chi} \otimes H^{0}\left(Z, \Omega_{Z}^{n}\right)^{\chi^{*}}
$$


In order to get counterexamples for the infinitesimal Torelli theorem for periods of $n$-forms, we have to look for situations where, although

$$
\bigoplus_{\chi \in G^{*}} H^{n-1}\left(Z, \Omega_{Z}^{1}\right)^{\chi} \otimes H^{0}\left(Z, \Omega_{Z}^{n}\right)^{\chi^{*}} \rightarrow\left(H^{n-1}\left(Z, \Omega_{Z}^{1} \otimes \Omega_{Z}^{n-1}\right)\right)^{G}
$$

is surjective, still

$$
H^{n-1}\left(Z, \Omega_{Z}^{1}\right)^{G} \otimes H^{0}\left(Z, \Omega_{Z}^{n}\right)^{G} \rightarrow H^{n-1}\left(Z, \Omega_{Z}^{1} \otimes \Omega_{Z}^{n-1}\right)^{G}
$$

fails to be surjective.

The first case where this occurs is the one of a classical Godeaux surface. This is the quotient of a smooth quintic $Z$ in $\mathbb{P}^{3}$ on which $G:=\mathbb{Z} / 5$ acts freely. In this case however $H^{0}\left(\Omega_{Z}^{2}\right)^{G}=0$, whence there is no period map.

We tried to cook up other examples as quotients, e.g. of complete intersections in projective space, but the examples which worked best were the cases of quotients of products of curves. For these we will produce examples where the canonical bundle remains ample or even quasi very ample, but infinitesimal Torelli fails.

2. Surfaces isogenous to a product. Let us recall the notion of surfaces isogenous to a higher product (prop. 3.11 of [Cat00] ensures that the following two properties 1) and 2) of a surface are equivalent).

Definition 2.1. A surface $S$ is said to be isogenous to a higher product if and only if, equivalently, either

1) $S$ admits a finite unramified covering which is isomorphic to a product of curves of genera at least two, or

2) $S$ is a quotient $S:=\left(C_{1} \times C_{2}\right) / G$, where the $C_{i}$ 's are curves of genus least two, and $G$ is a finite group acting freely on $Z:=\left(C_{1} \times C_{2}\right)$.

We have two cases: the mixed case, where the action of $G$ exchanges the two factors (and then $C_{1}, C_{2}$ are isomorphic), and the unmixed case where $G$ acts via a product action.

We shall assume throughout that we have such a surface and that we are in the unmixed case, thus we have a finite group $G$ acting on two curves $C_{1}, C_{2}$ with genera $g_{1}, g_{2} \geq 2$, and acting freely by the product action on $Z:=C_{1} \times C_{2}$. We will now examine the infinitesimal Torelli map of the quotient $S:=C_{1} \times C_{2} / G$.

In the following theorem we shall use the standard notation: given a finite group $G$, we let $G^{*}$ be the set of characters of irreducible representations of $G$, and, for $\chi \in G^{*}$ and $V$ a $G$-representation, we denote by $V^{\chi}$ the $\chi$-isotypical component. Finally, for $\chi \in G^{*}, \chi^{*}$ denotes the character of the dual irreducible representation.

TheOrem 2.2. Let $G, C_{1}, C_{2}$ and $S$ be as above, and assume that $G$ is abelian.

Then the infinitesimal Torelli theorem holds for $S$, i.e., we have the surjectivity of the following linear map

$$
d \Phi_{2}^{*}: H^{1}\left(S, \Omega_{S}^{1}\right) \otimes H^{0}\left(S, \Omega_{S}^{2}\right) \longrightarrow H^{1}\left(S, \Omega_{S}^{1} \otimes \Omega_{S}^{2}\right),
$$

if and only if the two maps

$$
\bigoplus_{\chi \in G^{*}: H^{0}\left(C_{2}, \Omega_{C_{2}}^{1}\right) \chi \neq 0} H^{0}\left(C_{1}, \Omega_{C_{1}}^{1}\right)^{\chi} \otimes H^{0}\left(C_{1}, \Omega_{C_{1}}^{1}\right)^{\chi^{*}} \longrightarrow H^{0}\left(C_{1}, \Omega_{C_{1}}^{1} \otimes \Omega_{C_{1}}^{1}\right)^{G}
$$


and

$$
\bigoplus_{\chi \in G^{*}: H^{0}\left(C_{1}, \Omega_{C_{1}}^{1}\right) \chi \neq 0} H^{0}\left(C_{2}, \Omega_{C_{2}}^{1}\right)^{\chi} \otimes H^{0}\left(C_{2}, \Omega_{C_{2}}^{1}\right)^{\chi^{*}} \longrightarrow H^{0}\left(C_{2}, \Omega_{C_{2}}^{1} \otimes \Omega_{C_{2}}^{1}\right)^{G}
$$

are both surjective.

Proof. Let $Z:=C_{1} \times C_{2}$ and denote the two projections to $C_{1}$ resp. $C_{2}$ by $p_{1}$, resp. $p_{2}$. Then $\Omega_{Z}^{1}=p_{1}^{*} \Omega_{C_{1}}^{1} \oplus p_{2}^{*} \Omega_{C_{2}}^{1}$. Therefore we obtain

$$
\begin{gathered}
H^{1}\left(S, \Omega_{S}^{1}\right)=H^{1}\left(Z, \Omega_{Z}^{1}\right)^{G}=H^{1}\left(Z,\left(\Omega_{C_{1}}^{1} \otimes \mathcal{O}_{C_{2}}\right) \oplus\left(\mathcal{O}_{C_{1}} \otimes \Omega_{C_{2}}^{1}\right)\right)^{G}= \\
\left(H^{1}\left(C_{1}, \Omega_{C_{1}}^{1}\right) \otimes H^{0}\left(C_{2}, \mathcal{O}_{C_{2}}\right)\right) \oplus\left(H^{0}\left(C_{1}, \mathcal{O}_{C_{1}}\right) \otimes H^{1}\left(C_{2}, \Omega_{C_{2}}^{1}\right) \oplus\right. \\
\bigoplus_{\chi \in G^{*}}\left(H^{0}\left(C_{1}, \Omega_{C_{1}}^{1}\right)^{\chi} \otimes H^{1}\left(C_{2}, \mathcal{O}_{C_{2}}\right)^{\chi^{*}}\right) \oplus \bigoplus_{\chi \in G^{*}}\left(H^{1}\left(C_{1}, \mathcal{O}_{C_{1}}\right)^{\chi} \otimes H^{0}\left(C_{2}, \Omega_{C_{2}}^{1}\right)^{\chi^{*}}\right) .
\end{gathered}
$$

Here, we use the convenient notation for the external tensor product of coherent sheaves

$$
\mathcal{F} \otimes \mathcal{G}=p_{1}^{*} \mathcal{F} \otimes p_{2}^{*} \mathcal{G}
$$

We remark that the above holds by Künneth's formula and Schur's lemma. Moreover, we use that $H^{1}\left(C_{1}, \Omega_{C_{1}}^{1}\right)$ and $H^{1}\left(C_{2}, \Omega_{C_{2}}^{1}\right)$ are automatically invariant, since every automorphism of $C_{i}$ maps the fundamental class to itself.

Again, using Schur's lemma we get

$$
\begin{gathered}
H^{0}\left(S, \Omega_{S}^{2}\right)=H^{0}\left(Z, \Omega_{Z}^{2}\right)^{G}=H^{0}\left(Z, \Omega_{C_{1}}^{1} \otimes \Omega_{C_{2}}^{1}\right)^{G}= \\
\bigoplus_{\chi \in G^{*}} H^{0}\left(C_{1}, \Omega_{C_{1}}^{1}\right)^{\chi} \otimes H^{0}\left(C_{2}, \Omega_{C_{2}}^{1}\right)^{\chi^{*}} .
\end{gathered}
$$

Moreover, we have

$$
\begin{gathered}
H^{1}\left(S, \Omega_{S}^{1} \otimes \Omega_{S}^{2}\right)=H^{1}\left(Z, \Omega_{Z}^{1} \otimes \Omega_{Z}^{2}\right)^{G}= \\
H^{1}\left(Z,\left(\Omega_{C_{1}}^{1}\right)^{2} \otimes \Omega_{C_{2}}^{1}\right)^{G} \oplus H^{1}\left(Z, \Omega_{C_{1}}^{1} \otimes\left(\Omega_{C_{2}}^{1}\right)^{2}\right)^{G}= \\
\left(H^{1}\left(C_{1},\left(\Omega_{C_{1}}^{1}\right)^{2}\right) \otimes H^{0}\left(C_{2}, \Omega_{C_{2}}^{1}\right)\right)^{G} \oplus\left(H^{0}\left(C_{1},\left(\Omega_{C_{1}}^{1}\right)^{2}\right) \otimes H^{1}\left(C_{2}, \Omega_{C_{2}}^{1}\right)\right)^{G} \\
\oplus\left(H^{1}\left(C_{1}, \Omega_{C_{1}}^{1}\right) \otimes H^{0}\left(C_{2},\left(\Omega_{C_{2}}^{1}\right)^{2}\right)\right)^{G} \oplus\left(H^{0}\left(C_{1}, \Omega_{C_{1}}^{1}\right) \otimes H^{1}\left(C_{2},\left(\Omega_{C_{2}}^{1}\right)^{2}\right)\right)^{G} .
\end{gathered}
$$

Using now the fact that

$$
H^{1}\left(C_{i},\left(\Omega_{C_{i}}^{1}\right)^{2}\right)=0, \quad H^{1}\left(C_{i}, \Omega_{C_{i}}^{1}\right)=H^{1}\left(C_{i}, \Omega_{C_{i}}^{1}\right)^{G} \quad \text { for } i=1,2
$$

we obtain the simpler expression

$$
H^{1}\left(S, \Omega_{S}^{1} \otimes \Omega_{S}^{2}\right)=
$$




$$
\begin{gathered}
\left(H^{0}\left(C_{1}, \mathcal{O}_{C_{1}}\left(2 K_{C_{1}}\right)\right)^{G} \otimes H^{1}\left(C_{2}, \mathcal{O}_{C_{2}}\left(K_{C_{2}}\right)\right) \oplus\right. \\
\oplus\left(H^{1}\left(C_{1}, \mathcal{O}_{C_{1}}\left(K_{C_{1}}\right)\right) \otimes H^{0}\left(C_{2}, \mathcal{O}_{C_{2}}\left(2 K_{C_{2}}\right)\right)^{G}\right) .
\end{gathered}
$$

By the non-degeneracy of the Serre duality, $\forall \chi \in G^{*}$ such that $H^{0}\left(C_{i}, \Omega_{C_{i}}^{1}\right)^{\chi} \neq 0$, $H^{0}\left(C_{i}, \Omega_{C_{i}}^{1}\right)^{\chi} \otimes H^{1}\left(C_{i}, \mathcal{O}_{C_{i}}\right)^{\chi^{*}} \rightarrow H^{1}\left(C_{i}, \Omega_{C_{i}}^{1}\right)$ is onto, whence we conclude that the dual Torelli map

$$
d \Phi_{2}^{*}: H^{1}\left(S, \Omega_{S}^{1}\right) \otimes H^{0}\left(S, \Omega_{S}^{2}\right) \longrightarrow H^{1}\left(S, \Omega_{S}^{1} \otimes \Omega_{S}^{2}\right)
$$

is surjective if and only if the two maps

$$
\bigoplus_{\chi \in G^{*}: H^{0}\left(C_{2}, \Omega_{C_{2}}^{1}\right) \chi \neq 0} H^{0}\left(C_{1}, \Omega_{C_{1}}^{1}\right)^{\chi} \otimes H^{0}\left(C_{1}, \Omega_{C_{1}}^{1}\right)^{\chi^{*}} \longrightarrow H^{0}\left(C_{1}, \Omega_{C_{1}}^{1} \otimes \Omega_{C_{1}}^{1}\right)^{G}
$$

and

$$
\bigoplus_{\chi \in G^{*}: H^{0}\left(C_{1}, \Omega_{C_{1}}^{1}\right) \chi \neq 0} H^{0}\left(C_{2}, \Omega_{C_{2}}^{1}\right)^{\chi} \otimes H^{0}\left(C_{2}, \Omega_{C_{2}}^{1}\right)^{\chi^{*}} \longrightarrow H^{0}\left(C_{2}, \Omega_{C_{2}}^{1} \otimes \Omega_{C_{2}}^{1}\right)^{G}
$$

are both surjective.

We assume now that $G$ is a cyclic group of order $d$. Let $C_{1}$ and $C_{2}$ be smooth curves such that

1) $G$ acts freely on $C_{1}$,

2) $G$ acts on $C_{2}$ in such a way that $C_{2} / G \cong \mathbb{P}^{1}$.

Then $G$ acts freely on $Z:=C_{1} \times C_{2}$.

Proposition 2.3. Assume that $C_{1}, C_{2}$ are as above, and let $C_{1}^{\prime}:=C_{1} / G$.

For $G=\mathbb{Z} / d$ the infinitesimal Torelli theorem does not hold for $S:=\left(C_{1} \times C_{2}\right) / G$, if, $g^{\prime}$ being the genus of $C_{1}^{\prime}$,

- $d=2$ and $2 \leq g^{\prime} \leq 5$

- $d=3$ and $g^{\prime}=3$

- $3 \leq d \leq 5$ and $g^{\prime}=2$.

Proof. As we have seen in theorem 2.2 it suffices to show that

$$
\Phi: \bigoplus_{\chi \in G^{*}: H^{0}\left(C_{2}, \Omega_{C_{2}}^{1}\right)^{\chi} \neq 0} H^{0}\left(C_{1}, \Omega_{C_{1}}^{1}\right)^{\chi} \otimes H^{0}\left(C_{1}, \Omega_{C_{1}}^{1}\right)^{\chi^{*}} \longrightarrow H^{0}\left(C_{1}, \Omega_{C_{1}}^{1} \otimes \Omega_{C_{1}}^{1}\right)^{G}
$$

is not surjective.

Let us fix an isomorphism of $G$ with the group of $d$-th roots of unity, and let us then denote by $H^{0}\left(C_{1}, \mathcal{O}_{C_{1}}\left(K_{C_{1}}\right)\right)^{i}$ the eigenspace of $H^{0}\left(C_{1}, \mathcal{O}_{C_{1}}\left(K_{C_{1}}\right)\right)$ belonging to the character $i \in(\mathbf{Z} / d)$. We have

$$
H^{0}\left(C_{1}, \mathcal{O}_{C_{1}}\left(K_{C_{1}}\right)\right)^{i}=H^{0}\left(C_{1}^{\prime}, \mathcal{O}_{C_{1}^{\prime}}\left(K_{C_{1}^{\prime}}\right) \otimes \mathcal{L}^{i}\right),
$$


where $\mathcal{L} \in \operatorname{Pic}\left(C_{1}^{\prime}\right)$ is an element of precisely $d$ - torsion, whence

$$
\operatorname{dim} H^{0}\left(C_{1}, \mathcal{O}_{C_{1}}\left(K_{C_{1}}\right)\right)^{i}=g^{\prime}-1 .
$$

Therefore we have

$$
\operatorname{dim} \Phi\left(\bigoplus_{\chi \in G^{*}: H^{0}\left(C_{2}, \Omega_{C_{2}}^{1}\right)} H^{0}\left(C_{1}, \Omega_{C_{1}}^{1}\right)^{\chi} \otimes H^{0}\left(C_{1}, \Omega_{C_{1}}^{1}\right)^{\chi^{*}}\right)=
$$

$\operatorname{dim} \Phi\left(\bigoplus_{\chi \neq 0} H^{0}\left(C_{1}, \Omega_{C_{1}}^{1}\right)^{\chi} \otimes H^{0}\left(C_{1}, \Omega_{C_{1}}^{1}\right)^{\chi^{*}}\right) \leq\left(\frac{d}{2}-1\right)\left(g^{\prime}-1\right)^{2}+\frac{g^{\prime}\left(g^{\prime}-1\right)}{2}$,

for $d$ even and

$$
\operatorname{dim} \Phi\left(\bigoplus_{\chi \neq 0} H^{0}\left(C_{1}, \Omega_{C_{1}}^{1}\right)^{\chi} \otimes H^{0}\left(C_{1}, \Omega_{C_{1}}^{1}\right)^{\chi^{*}}\right) \leq \frac{d-1}{2}\left(g^{\prime}-1\right)^{2},
$$

for $d$ odd.

Observe now that

$$
\operatorname{dim} H^{0}\left(C_{1}, \mathcal{O}_{C_{1}}\left(2 K_{C_{1}}\right)\right)^{G}=h^{0}\left(C_{1}^{\prime}, \mathcal{O}_{C_{1}^{\prime}}\left(2 K_{C_{1}^{\prime}}\right)\right)=3 g^{\prime}-3 .
$$

From this we see immediately that

$$
\operatorname{dim} \Phi\left(\bigoplus_{\chi \neq 0} H^{0}\left(C_{1}, \Omega_{C_{1}}^{1}\right)^{\chi} \otimes H^{0}\left(C_{1}, \Omega_{C_{1}}^{1}\right)^{\chi^{*}}\right)<\operatorname{dim} H^{0}\left(C_{1}, \mathcal{O}_{C_{1}}\left(2 K_{C_{1}}\right)\right)^{G}
$$

for

- $d=2$ and $2 \leq g^{\prime} \leq 5$

- $d=3$ and $g^{\prime}=3$

- $3 \leq d \leq 5$ and $g^{\prime}=2$.

We see here that the infinitesimal Torelli theorem fails simply for reasons of dimension. Also it is obvious from the formulae that we cannot get such an easy failure as soon as $g^{\prime}$ and $d$ become bigger.

REMARK 2.4. We have now constructed a series of counterexamples to the infinitesimal Torelli theorem. Of course we are now interested to see whether it is possible to obtain that $K_{S}$ be very ample. If $d=2$ there is no hope, since $\mathbf{Z} / 2$ induces on $C_{2}$ the hyperelliptic involution, so that the canonical map of $S$ is of degree $\geq 2$ : whence we obtain that $K_{S}$ is ample, but not very ample, or quasi very ample. Therefore we will concentrate on the second and third case.

Before constructing a concrete family of examples, we recall some of the notations and results from Pardini's article on abelian covers ([Pa91]):

Let $\pi: X \longrightarrow Y$ be a (finite) abelian cover with group $G$, where $Y$ is smooth and $X$ is a normal variety. Then $\pi$ is flat and the action of $G$ induces a splitting

$$
\pi_{*} \mathcal{O}_{X}=\bigoplus_{\chi \in G^{*}} L_{\chi}^{-1}
$$


where $G$ acts on $L_{\chi}^{-1}$ via the character $\chi$. The invariant summand $L_{0}$ is isomorphic to $\mathcal{O}_{Y}$. We denote by $D$ the branching divisor of $\pi$.

We denote by $\mathfrak{S}$ the set of cyclic subgroups of $G$ and for each $H \in \mathfrak{S}$ we denote by $S_{H}$ the set of generators of the group of characters $H^{*}$. Then we can write

$$
D=\sum_{H \in \mathfrak{S}} \sum_{\psi \in S_{H}} D_{H, \psi}
$$

where $D_{H, \psi}$ is the sum of all components of $D$ having inertia group $H$ and character $\psi$.

We recall that the inertia group $H$ of a component $T$ of the ramification divisor $R$ is defined by

$$
H=\{h \in G: h x=x, \forall x \in T\}
$$

Moreover, we associate to $T$ a generator $\psi_{T}$ of $H^{*}$, in fact there is a parameter $t$ of $\mathcal{O}_{X, T}$ such that the action of $H$ is given by

$$
h t=\psi_{T}(h) t, \quad \forall h \in H .
$$

Now, if $S$ is a component of $D$, then all the components of $\pi^{-1}(S)$ have the same inertia group and isomorphic representations on the cotangent space at the corresponding points of $X$ (since $G$ is abelian). So it makes sense to associate to every component of $D$ a cyclic subgroup $H$ of $G$ and a generator $\psi$ of $H^{*}$.

For every pair of characters $\chi, \chi^{\prime} \in G^{*}$, for every $H \in \mathfrak{S}$ and for every $\psi \in S_{H}$ we can write

$$
\chi\left|H=\psi^{i_{\chi}}, \quad \chi^{\prime}\right| H=\psi^{i_{\chi^{\prime}}}, \quad i_{\chi}, i_{\chi^{\prime}} \in\left\{0, \ldots, m_{H}-1\right\},
$$

where $m_{H}$ is the order of $H$. One sets $\epsilon_{\chi, \chi^{\prime}}^{H, \psi}:=0$, if $i_{\chi}+i_{\chi^{\prime}}<m_{H}$ and $=1$ otherwise.

Definition 2.5. Let $\pi: X \longrightarrow Y$ be an abelian cover with group $G$. Moreover, assume that $X$ is normal and $Y$ smooth. Then the sheaves $L_{\chi}, \chi \in G^{*}$, and the divisors $D_{H, \psi}$ are called the building data of the covering.

Now we are ready to formulate the following result of R. Pardini (cf. [Pa91], theorem 2.1).

TheOrem 2.6. (PARDINI) Let $G$ be a finite abelian group.

1) Let $\pi: X \longrightarrow Y$ be a covering with group $G$, where $X$ is a normal variety and $Y$ is smooth and complete. Then the building data of $\pi$ satisfy the following linear equivalences:

$$
L_{\chi}+L_{\chi^{\prime}} \equiv L_{\chi \chi^{\prime}}+\sum_{H \in \mathfrak{S}} \sum_{\psi \in S_{H}} \epsilon_{\chi, \chi^{\prime}}^{H,{ }^{\prime}} D_{H, \psi_{H}}
$$

2) Given any set of data $L_{\chi}, D_{H, \psi}$ satisfying the above linear equivalences, there is a unique (up to isomorphisms of Galois covers) abelian cover $\pi: X \longrightarrow Y$ such that $L_{\chi}, D_{H, \psi}$ are its building data, if $X$ is normal.

Sometimes, however, we shall also specify an abelian cover through the normalization of a singular abelian cover. 
For instance, let $P_{1}, \ldots, P_{5}$ be five pairwise different points in $\mathbb{P}^{1}$ and let $L$ be a divisor on $\mathbb{P}^{1}$ such that

$$
3 L \equiv P_{1}+P_{2}+P_{3}+P_{4}+2 P_{5} \equiv \mathcal{O}_{\mathbb{P}^{1}}(6) .
$$

Then there is a unique $\mathbb{Z} / 3$ - covering $\pi: C_{2} \longrightarrow \mathbb{P}^{1}$ with branch locus $P_{1}+P_{2}+P_{3}+$ $P_{4}+2 P_{5}$. We remark that by Hurwitz' formula the genus of $C_{2}$ has to be 3 .

We calculate now the decomposition of $H^{0}\left(C_{2}, \Omega_{C_{2}}^{1}\right)$ into eigenspaces according to the characters of $G$. We denote the characters of $G=\mathbb{Z} / 3$ simply by $0,1,2$.

Using the above theorem we can now calculate $L_{\chi}$. First of all, $L_{0}=\mathcal{O}_{\mathbb{P} 1}$ and $L_{1}=\mathcal{O}_{\mathbb{P}^{1}}(2)$. Using the above formula we obtain $L_{2}=L_{1}+L_{1}-P_{5}=\mathcal{O}_{\mathbb{P}^{1}}(3)$. By [Pa91], prop. 4.1, we know that

$$
\left(\pi_{*} \Omega_{C_{2}}^{1}\right)^{\chi}=\Omega_{\mathbb{P}^{1}}^{1} \otimes L_{\chi^{-1}} .
$$

Therefore we conclude that

$$
\begin{gathered}
H^{0}\left(\Omega_{C_{2}}^{1}\right)=H^{0}\left(\Omega_{C_{2}}^{1}\right)^{0} \oplus H^{0}\left(\Omega_{C_{2}}^{1}\right)^{1} \oplus H^{0}\left(\Omega_{C_{2}}^{1}\right)^{2}= \\
H^{0}\left(\Omega_{\mathbb{P}^{1}}^{1}\right) \oplus H^{0}\left(\Omega_{\mathbb{P}^{1}}^{1} \otimes \mathcal{O}(3)\right) \oplus H^{0}\left(\Omega_{\mathbb{P}^{1}}^{1} \otimes \mathcal{O}(2)\right)= \\
H^{0}\left(\mathbb{P}^{1}, \mathcal{O}(1)\right) \oplus H^{0}\left(\mathbb{P}^{1}, \mathcal{O}\right) .
\end{gathered}
$$

THEOREM 2.7. Let $C_{1}^{\prime}$ be a general curve of genus 3 and let $C_{1}$ be a connected unramified covering of degree 3. Moreover, let $C_{2}$ be as above. Then the following holds:

1) $G:=\mathbb{Z} / 3$ operates freely on $C_{1} \times C_{2}$;

2) infinitesimal Torelli fails for $S:=C_{1} \times C_{2} / G$;

3) $K_{S}$ is quasi very ample, i.e., it gives a birational morphism which is a local embedding on the complement of a finite set.

Proof. We observe that, by proposition 2.3, we only have to show part 3 ).

For this we remark that $H^{0}\left(C_{1}, \Omega_{C_{1}}^{1}\right)^{0}$ has dimension 3 while $h^{0}\left(C_{1}, \Omega_{C_{1}}^{1}\right)^{1}=$ $h^{0}\left(C_{1}, \Omega_{C_{1}}^{1}\right)^{2}=2$.

Let $\eta$ be a 3 - torsion element in $\operatorname{Pic}\left(C_{1}^{\prime}\right)$ corresponding to the unramified covering $C_{1} \rightarrow C_{1}^{\prime}$.

Let $s_{1}, s_{2} \in H^{0}\left(C_{1}, \Omega_{C_{1}}^{1}\right)^{1}=H^{0}\left(C_{1}^{\prime}, K_{C_{1}^{\prime}}+\eta\right)$ and $t_{1}, t_{2} \in H^{0}\left(C_{1}, \Omega_{C_{1}}^{1}\right)^{2}=$ $H^{0}\left(C_{1}^{\prime}, K_{C_{1}^{\prime}}+2 \eta\right)$ yield respective bases of these vector spaces.

Let furthermore $\sigma_{1}, \sigma_{2} \in H^{0}\left(\Omega_{C_{2}}^{1}\right)^{1}$, resp. $\sigma_{3} \in H^{0}\left(\Omega_{C_{2}}^{1}\right)^{2}$ yield respective bases of these vector spaces. It is easy to see that the divisor of zeroes of $\sigma_{3}$ is the sum $Q_{1}+Q_{2}+Q_{3}+Q_{4}$, where $Q_{i}$ is the point lying over $P_{i}$.

Instead the linear series cut by $\left|\lambda_{1} \sigma_{1}+\lambda_{2} \sigma_{2}=0\right|$ has $Q_{5}$ as base point, and then yields residually the $g_{3}^{1}$ which gives the triple covering of $\mathbb{P}^{1}$. It follows immediately that the curve $C_{2}$ is not hyperelliptic, whence its canonical system is very ample. Observe then that

$$
\left\{s_{1} \otimes \sigma_{3}, s_{2} \otimes \sigma_{3}, t_{1} \otimes \sigma_{1}, t_{2} \otimes \sigma_{2}, t_{1} \otimes \sigma_{2}, t_{2} \otimes \sigma_{1}\right\}
$$

is a basis of $H^{0}\left(S, \mathcal{O}\left(K_{S}\right)\right)$. 
We will use the following

LEMMA 2.8. Let $C_{1}^{\prime}$ be a generic, (in particular, non hyperelliptic) curve of genus 3 and let $\eta$ be a non trivial 3- torsion element of Pic $\left(C_{1}^{\prime}\right)$. Then

1) the linear system $\left|K_{C_{1}^{\prime}}+\eta\right|$ is base point free,

2) the morphism $f: C_{1}^{\prime} \rightarrow \mathbb{P}^{1} \times \mathbb{P}^{1}$ induced by the product of the two pencils $\mid K_{C_{1}^{\prime}}+$ $\eta \mid$ and $\left|K_{C_{1}^{\prime}}+2 \eta\right|$ is birational onto its image, which is a curve $\Gamma$ of bidegree $(4,4)$ having exactly 6 ordinary double points as singularities, on the set $\mathbb{M}:=\{(x, y) \mid x \neq$ $y, x, y \in\{0,1, \infty\}\}$.

Let's postpone the proof of the lemma and infer from it the quasi very ampleness of the canonical system of $S$.

We observe first of all that the canonical system $K_{S}$ is base point free.

In fact, we can apply Lemma 2.8 twice, for $\eta$ and for $2 \eta$, so that, given a point $(x, y) \in C_{1} \times C_{2}$ we may assume (after a change of basis) $s_{2}, t_{2}$ not to vanish on the image of $x$ in $C_{1}^{\prime}$. And since the quotient of $C_{2}$ is rational, $H^{0}\left(C_{2}, \Omega_{C_{2}}^{1}\right)^{0}=0$ thus, the canonical system of a curve being base point free, for each choice of $y$ there is a section $\sigma_{j}$, for some $1 \leq j \leq 3$, which does not vanish on $y$.

Let us now take two points $(x, y),\left(x^{\prime \prime}, y^{\prime \prime}\right) \in C_{1} \times C_{2}$ representing two distinct points in $S$, and let us assume that they are not separated by the canonical map of $S$.

If $x=x^{\prime \prime}$, then the two points are separated since $\sigma_{1}, \sigma_{2}, \sigma_{3}$ yield the canonical map of $C_{2}$, which is non hyperelliptic.

If $y=Q_{5}$ and the two points are not separated, it must be also $y^{\prime \prime}=Q_{5}$, since the last 4 coordinates are then equal to zero.

But then $\left(s_{1}(x): s_{2}(x)\right)=\left(s_{1}\left(x^{\prime \prime}\right): s_{2}\left(x^{\prime \prime}\right)\right)$, which means that $x^{\prime \prime}$ and $x$ map to respective points $z^{\prime \prime}$ and $z$ such that $z^{\prime \prime}+z$ is contained in a divisor of $\left|K_{C_{1}^{\prime}}+\eta\right|$. Since we have two distinct points of $S, z^{\prime \prime} \neq z$. We get therefore a singular line in the canonical image $\phi_{K}(S)$, which is a quadruple line.

Assume now $y=Q_{j}, 1 \leq j \leq 4$ : then it must also be $y^{\prime \prime}=Q_{i}, 1 \leq i \leq 4$ since the first two coordinates are zero. If however $i \neq j$, then we may assume without loss of generality $\sigma_{1}\left(Q_{j}\right)=0, \sigma_{1}\left(Q_{i}\right) \neq 0$, a contradiction.

It follows that $y^{\prime \prime}=y=Q_{j}, 1 \leq j \leq 4$, and we conclude as in the previous case that $x^{\prime \prime}$ and $x$ map to respective points $z^{\prime \prime} \neq z$ such that $z^{\prime \prime}, z$ have the same image point under $\left|K_{C_{1}^{\prime}}+2 \eta\right|$. We get thus 4 more quadruple lines in the canonical image $\phi_{K}(S)$.

We assume now that $y^{\prime \prime}, y$ are no ramification points $\left(y^{\prime \prime}, y \neq Q_{j}, 1 \leq j \leq 5\right)$. We may assume also as before that $\sigma_{1}(y) \neq 0, \sigma_{2}(y)=0$. We infer then immediately that $y^{\prime \prime}$ is in the $G$-orbit of $y$.

Thus, without loss of generality, and by the first step, we may assume $y^{\prime \prime}=y$ and that the respective image points $z, z^{\prime \prime}$ of $x, x^{\prime \prime}$ in $C_{1}$ are distinct.

Since $\sigma_{1}(y) \neq 0, \sigma_{3}(y) \neq 0$, it follows that it must be $f(z)=f\left(z^{\prime \prime}\right)$. Fix then one of the 54 pairs of points $x, x^{\prime \prime}$ with the property that $z \neq z^{\prime \prime}$, but $f(z)=f\left(z^{\prime \prime}\right)$.

We ask whether for $y \in C_{2}$ the points $(x, y),\left(x^{\prime \prime}, y\right)$ are not separated. Again after a change of basis, since $f(z)=f\left(z^{\prime \prime}\right)$, we may assume $s_{1}(x)=s_{1}\left(x^{\prime \prime}\right)=t_{1}(x)=$ $t_{1}\left(x^{\prime \prime}\right)=0$. Then the question is whether $\left(s_{2}(x): t_{2}(x)\right)=\left(s_{2}\left(x^{\prime \prime}\right): t_{2}\left(x^{\prime \prime}\right)\right)$. In other words, the question we must answer is whether the subseries of the canonical system of $C_{1}$, given by the 4 sections $s_{1}, s_{2}, t_{1}, t_{2}$ separates the two points $x, x^{\prime \prime}$.

We use here the fact that the point $f(z)=f\left(z^{\prime \prime}\right)$ is an ordinary double point, i.e., with distinct tangents. There are local coordinates $(u, v)$ on $\mathbb{P}^{1} \times \mathbb{P}^{1}$ such that 
$f(z)=f\left(z^{\prime \prime}\right)$ corresponds to the origin. We show in the proof of lemma 2.8 that locally the triple cover is obtained by extracting the third root of $u / v, w^{3}=u / v$.

Accordingly, the 4 sections $s_{1}, s_{2}, t_{1}, t_{2}$ are locally expressed by $1, u, w, w v$. If $w(x)=w\left(x^{\prime \prime}\right)$, then we would have $(u / v)(z)=(u / v)\left(z^{\prime \prime}\right)$, a contradiction.

We have thus proven that for $C_{1}^{\prime}$ generic the canonical system of $S$ yields a morphism which is injective except for the curves images of $C_{1} \times\left\{Q_{j}\right\}, 1 \leq j \leq 5$.

There remains to prove that the canonical map is a local embedding except at finitely many points.

Lemma 2.8 shows that for a general curve $C$ of genus 3, the mapping $f: C \rightarrow$ $\mathbb{P}^{1} \times \mathbb{P}^{1}$, corresponding to the sum of the two linear systems $\left|K_{C}+\eta\right|$ and $\left|K_{C}+2 \eta\right|$, is everywhere a local embedding.

We will prove that the canonical system $\left|K_{S}\right|$ separates tangent vectors at a point corresponding to $(x, y) \in C_{1} \times C_{2}$, unless $y$ is a ramification point for the triple cover of $\mathbb{P}^{1}$, and $x$ is a ramification point for one of the two $g_{4}^{1}$ 's $\left|K_{C}+\eta\right|$ and $\left|K_{C}+2 \eta\right|$.

Without loss of generality we may then take a point $(x, y) \in C_{1} \times C_{2}$ and we assume $s_{1}, t_{1}$ to vanish on $x$ and $s_{2}, t_{2}$ not to vanish on $x$.

We look for two sections yielding two curves which are smooth at $(x, y)$ and have distinct tangents.

If we have that $s_{1}$ vanishes of order exactly 1 at $x$ and $\sigma_{3}(y) \neq 0$, we are done, since there is always a section $\sigma_{i}$ vanishing simply at $y$, and $s_{1} \sigma_{3}, s_{2} \sigma_{i}$ give two curves with vertical, respectively horizontal tangent.

Similarly, if $t_{1}$ vanishes of order exactly 1 at $x$, and $y \neq Q_{5}$, then we may assume $\sigma_{1}(y) \neq 0$, and $\sigma_{2}$ to vanish simply on $y$, unless we are in a branch point $Q_{i}$. For $i \leq 4$, however, $\sigma_{3}$ vanishes simply and we are therefore done. $\square$ for Theorem 2.7

Proof of lemma 2.8. The moduli space of curves of genus 3 has dimension 6 and the hyperelliptic curves form a five dimensional algebraic subset. Hence we can suppose that $C_{1}^{\prime}$ is not hyperelliptic. Therefore $C_{1}^{\prime}$ is canonically embedded as a plane quartic in $\mathbb{P}^{2}$. Let $\eta$ be an element of $\operatorname{Pic}\left(C_{1}^{\prime}\right)_{3}$. We note that $P$ is a base point of the linear system $\left|K_{C_{1}^{\prime}}+\eta\right|$ if and only if

$$
H^{0}\left(C_{1}^{\prime}, \mathcal{O}(K+\eta)\right)=H^{0}\left(C_{1}^{\prime}, \mathcal{O}(K+\eta-P)\right) .
$$

Since $\operatorname{dim} H^{0}\left(C_{1}^{\prime}, \mathcal{O}(K+\eta)\right)=2$ this is equivalent to

$$
\operatorname{dim} H^{1}\left(C_{1}^{\prime}, \mathcal{O}(K+\eta-P)\right)=1 .
$$

Since $H^{1}\left(C_{1}^{\prime}, \mathcal{O}(K+\eta-P)\right) \cong H^{0}\left(C_{1}^{\prime}, \mathcal{O}(P-\eta)\right)^{*}$, it follows that there is a point $P^{\prime}$ such that $P-\eta \equiv P^{\prime}$. Therefore $3 P \equiv 3 P^{\prime}$. By Riemann - Roch we have

$$
\begin{gathered}
\operatorname{dim} H^{0}\left(C_{1}^{\prime}, \mathcal{O}(K-3 P)\right)= \\
\operatorname{deg}(K-3 P)+1-g\left(C_{1}^{\prime}\right)+\operatorname{dim}^{1}\left(C_{1}^{\prime}, \mathcal{O}(K-3 P)=1+1-3+2=1,\right.
\end{gathered}
$$

and there is a point $Q$ such that $Q \equiv K-3 P \equiv K-3 P^{\prime}$.

Geometrically this means that, considering $C_{1}^{\prime}$ as a plane quartic, $C_{1}^{\prime}$ has two inflection points $P, P^{\prime}$, such that the tangent lines to these points intersect in $Q \in C_{1}^{\prime}$. Let now $p, q, p^{\prime}$ be three non collinear points in $\mathbb{P}^{2}$. Then the dimension of the group of automorphisms of $\mathbb{P}^{2}$ leaving the three points fixed has dimension 2. The quartics in $\mathbb{P}^{2}$ form a linear system of dimension 14 . Imposing that a plane quartic contains 
the point $q$ is one linear condition. Moreover, the condition that the line containing $p$ and $q$ is a tritangent to the quartic gives further three linear conditions as well as the condition that the line containing $p^{\prime}$ and $q$ is a tritangent to the quartic. Therefore the linear subsystem of quartics $C$ having two inflection points $P, P^{\prime}$, such that the tangent lines to these points intersect in $Q \in C$ has dimension $14-2-3-3-1=5$, whence they are special.

Part 1) is therefore proven and we set for convenience of notation $C:=C_{1}^{\prime}$. By 1) we may assume that both linear systems $\left|K_{C}+\eta\right|$ and $\left|K_{C}+2 \eta\right|$ are base point free.

Even if not strictly needed for our purposes, we try to describe how one reaches the conclusion that the curve $C$ must be birational to a curve $\Gamma \subset \mathbb{P}^{1} \times \mathbb{P}^{1}$ enjoying the properties stated in 2).

More generally, for $L \in P i c^{0}(C), L$ general, we obtain a morphism $f_{L}: C \rightarrow$ $\mathbb{P}^{1} \times \mathbb{P}^{1}$ corresponding to the sum of linear systems $\left|K_{C}+L\right|$ and $\left|K_{C}-L\right|$.

Set $\Gamma:=f_{L}(C)$. Then either $\Gamma$ is a curve of bidegree $(4,4)$, or $\operatorname{deg}\left(f_{L}\right)=2$ and $\Gamma$ is a curve of bidegree $(4,4)$, since if $\operatorname{deg}\left(f_{L}\right)=4$ then $L \equiv-L$ and $L$ is thus of 2-torsion.

We will assume that $f_{L}$ is birational, else $C$ is either hyperelliptic, or a double cover of an elliptic curve, which is then special because it is branched in 4 points, and we get only a family of dimension 5 in the moduli space.

Let $P_{1}, \ldots P_{m}$ be the (possibly infinitely near) singular points of $\Gamma$.

Then, $H_{1}, H_{2}$ being the respective divisors of a vertical, and of a horizontal line in $\mathbb{P}^{1} \times \mathbb{P}^{1}, \Gamma \in\left|4 H_{1}+4 H_{2}-\Sigma_{i=1, \ldots m} r_{i} P_{i}\right|$.

By adjunction, the canonical system of $C$ is cut by the series $\mid 2 H_{1}+2 H_{2}-$ $\Sigma_{i=1, \ldots m}\left(r_{i}-1\right) P_{i} \mid$.

Since $C$ has genus 3 , we obtain $\Sigma_{i=1, \ldots m} r_{i}\left(r_{i}-1\right)=12$. Whence, either $\Gamma$ has just an ordinary 4 -uple point or all the multiplicities are at most 3 .

In the former case, however, we take local coordinates $(u, v)$ at the 4 -uple point and see that the canonical map is given by $\left(u^{2} v: v^{2} u: u^{2} v^{2}\right)=(u: v: u v)$ and the curve $C$ is hyperelliptic.

In the latter case, we observe that $\Gamma$ lies on a regular surface, whence we have that the bicanonical system is obtained as the restriction of the linear system $\mid 4 H_{1}+$ $4 H_{2}-\Sigma_{i=1, \ldots m} 2\left(r_{i}-1\right) P_{i} \mid$ (in fact on the blown up surface $X$, we have $(-1)$ divisors $E_{i}$ and $H^{1}\left(\mathcal{O}_{X}\left(-\Sigma_{i=1, \ldots m}\left(2-r_{i}\right) E_{i}\right)\right)=0$ since $r_{i} \leq 3$, by Ramanujam's vanishing theorem).

We exploit at this point that $H_{1}$ pulls back to $K_{C}+L, H_{2}$ pulls back to $K_{C}-L$, thus there is a subseries of the bicanonical series cut by $H_{1}+H_{2}$. We infer the existence of a curve $G \in\left|3 H_{1}+3 H_{2}-\Sigma_{i=1, \ldots m} 2\left(r_{i}-1\right) P_{i}\right|$.

Assuming that $G$ is reduced, we see that $G$ has geometric genus $g(G)=4-$ $\Sigma_{i=1, \ldots m}\left(2 r_{i}-3\right)\left(r_{i}-1\right) P_{i} \leq-2$, and indeed $g(G) \leq-5$ if there is a triple point for $\Gamma$. Yet, $G$ has at most 6 components, thus $g(G) \geq-5$, and if equality holds, $G$ consists of 3 vertical and 3 horizontal lines, in particular it has no points of multiplicity 4 .

It follows then that all the $r_{i}=2$, and $G$ has three components, which are rational.

General case. $G=q_{1} \cup q_{2} \cup q_{3}$, where $q_{j}$ is of bidegree $(1,1)$, the curves $q_{i}$ and $q_{j}$ meet transversally in 2 points $P_{i, j}, P_{i, j}^{\prime}$. $\left.P_{i, j}^{\prime}\right) \mid$.

Fixing these three conics $q_{j}$, one takes a general curve $\Gamma \in \mid 4 H_{1}+4 H_{2}-\Sigma_{i, j} 2\left(P_{i, j}+\right.$

Let $X$ be the blow up of $\mathbb{P}^{1} \times \mathbb{P}^{1}$ in these 6 points, and let $\tilde{G}, \tilde{\Gamma}$ be the proper 
transforms of $G$, resp. $\Gamma$. By the exact sequence

$$
0 \rightarrow \mathcal{O}_{X}\left(H_{1}+H_{2}\right) \rightarrow \mathcal{O}_{X}(\tilde{\Gamma}) \rightarrow \mathcal{O}_{\tilde{G}}(\tilde{\Gamma}) \rightarrow 0
$$

we obtain $\operatorname{dim}|\tilde{\Gamma}|=4+3-1=6$. Since we have moreover $3+3+3$ moduli for the 3 conics $q_{j}$, after subtracting 6 moduli for the automorphisms of $\mathbb{P}^{1} \times \mathbb{P}^{1}$, we obtain a family of dimension $9=6+3$, which is the expected dimension, since 6 is the dimension of the moduli space of curves of genus 3 , and $3=\operatorname{dim} \operatorname{Pic}^{0}(C)$.

3 - Torsion case. Assume now that $L=\eta$ is a non trivial 3-torsion divisor: then $3 H_{i} \equiv 3 K_{C}$, whence we expect curves $\Delta_{1} \in\left|6 H_{1}+3 H_{2}-\Sigma_{i=1, \ldots m} 3\left(r_{i}-1\right) P_{i}\right|$, $\Delta_{2} \in\left|3 H_{1}+6 H_{2}-\Sigma_{i=1, \ldots m} 3\left(r_{i}-1\right) P_{i}\right|$. Intersecting on $X$ the proper transform of $\Delta_{i}$ with the proper transform of each component $q_{j}$ of $G$ we get intersection number $9-12=-3$, thus $G$ should be contained in $\Delta_{i}$.

But then $\Lambda_{1}:=\Delta_{1}-G, \Lambda_{2}:=\Delta_{2}-G$ provide divisors in $\left|3 H_{1}-\Sigma_{i=1, \ldots m}\left(r_{i}-1\right) P_{i}\right|$, respectively in $\left|3 H_{2}-\Sigma_{i=1, \ldots m}\left(r_{i}-1\right) P_{i}\right|$. Again, the intersection number of the proper transforms of $G$ and $\Lambda_{i}$ are negative $=6-12=-6$, thus we conclude that $G=\Lambda_{1}+\Lambda_{2}$, and that each $q_{j}$ is reducible.

The curve $\Gamma$ has 6 double points which lie on these 6 lines, but since the bidegree of $\Gamma$ is $(4,4)$ there are at most 2 singular points on each line, and without loss of generality, since we assumed $G$ to be reduced, we may assume that the 6 points are as in the statement of the lemma, namely, they are the points of the set $\mathbb{M}:=$ $\{(x, y) \mid x \neq y, x, y \in\{0,1, \infty\}\}$.

Claim 1. The general element $\Gamma \in\left|4 H_{1}+4 H_{2}-\mathbb{M}\right|$ is irreducible and has only ordinary double points as singularities.

Proof of claim 1. The general element has only double points as singularities, since we may just consider the subsystem $G+\left|H_{1}+H_{2}\right|$. Since the selfintersection of the proper transforms on $X$ of the curves in the linear system equals $32-24=8$, the system is not composed of a pencil, and the general member is irreducible. $\square$ for claim 1.

Claim 2. For any curve $\Gamma \in\left|4 H_{1}+4 H_{2}-\mathbb{M}\right|$ we get an unramified cyclic triple cover $Y$ of the normalization $C$ of $\Gamma$ by taking as $Y$ the normalization of the inverse image of $\Gamma$ in the cyclic triple cover $W$ of $\mathbb{P}^{1} \times \mathbb{P}^{1}$ branched on $G$, and such that $\Lambda_{1}$ is the divisor $D_{1}$ in Pardini's notation, $\Lambda_{2}$ is the divisor $D_{2}$.

Proof of claim 2. $G$ intersects $\Gamma$ only in the double points. Once we pull back the triple covering to $C$, for each point $p$ lying over a double point, the point $p$ appears in the branch divisor with multiplicity $1-1=0$. Thus $Y \rightarrow C$ is unramified. $\square$ for claim 2.

Final Observation. The linear system $\left|4 H_{1}+4 H_{2}-\mathbb{M}\right|$ has projective dimension equal to 6 , as previously indicated. We get therefore a 6 dimensional family, which is therefore dominant onto the moduli space of pairs $(C, \eta)$ as above.

REMARK 2.9. The family of surfaces considered in Theorem 2.7 has dimension 8.

We observe that the canonical image $\phi_{K}(S)$ has $S$ as its normalization. The inverse images of the singular curves of $\phi_{K}(S)$ are exactly the curves $C_{1} \times\left\{Q_{j}\right\}, 1 \leq$ $j \leq 5$. 
Therefore, the geometry of the canonical image $\phi_{K}(S)$ recovers $C_{1}$, which is the "missing" Hodge structure.

Replacing now the curve $C_{2}$ by a triple cover of $\mathbb{P}^{1}$ ramified in more points than in the previous example, we can produce an infinite series of examples of surfaces having quasi very ample canonical bundle for which the infinitesimal Torelli theorem fails. The construction goes as follows.

Let $k$ be any natural number bigger or equal to two and let $P_{1}, \ldots, P_{3 k-2}, P_{3 k-1}$ be pairwise different points in $\mathbb{P}^{1}$. We consider the triple cover $C_{k}$ of $\mathbb{P}^{1}$ ramified along

$$
P_{1}+\ldots+P_{3 k-2}+2 P_{3 k-1} .
$$

Then the genus of $C_{k}$ is equal to $3 k-3$. Using Pardini's formulae we can calculate the decomposition of $H^{0}\left(\Omega_{C_{2}}^{1}\right)$ into eigenspaces according to the characters of $G=\mathbb{Z} / 3$. We get: $L_{0}=\mathcal{O}_{\mathbb{P}^{1}}, L_{1}=\mathcal{O}_{\mathbb{P}^{1}}(k)$ and $L_{2}=\mathcal{O}_{\mathbb{P}^{1}}(2 k-1)$ and therefore

$$
\begin{gathered}
H^{0}\left(\Omega_{C_{k}}^{1}\right)=H^{0}\left(\Omega_{C_{k}}^{1}\right)^{0} \oplus H^{0}\left(\Omega_{C_{k}}^{1}\right)^{1} \oplus H^{0}\left(\Omega_{C_{k}}^{1}\right)^{2}= \\
H^{0}\left(\Omega_{\mathbb{P}^{1}}^{1}\right) \oplus H^{0}\left(\Omega_{\mathbb{P}^{1}}^{1} \otimes \mathcal{O}(2 k-1)\right) \oplus H^{0}\left(\Omega_{\mathbb{P}^{1}}^{1} \otimes \mathcal{O}(k)\right)= \\
H^{0}\left(\mathbb{P}^{1}, \mathcal{O}(2 k-3)\right) \oplus H^{0}\left(\mathbb{P}^{1}, \mathcal{O}(k-2)\right) .
\end{gathered}
$$

Using the same argument as in theorem 2.7 (note that we only use that the curve $C_{2}$ is not hyperelliptic and that $C_{2} / G=\mathbb{P}^{1}$ ) we obtain the following

TheORem 2.10. Let $k$ be any natural number bigger or equal to 2 and let $C_{1}^{\prime}$ be a general (non hyperelliptic) curve of genus 3 and let $C_{1}$ be a connected unramified covering of $C_{1}^{\prime}$ of degree 3 . Moreover, let $C_{k}$ be as above.

Then the following hold:

1) $G:=\mathbb{Z} / 3$ operates freely on $C_{1} \times C_{k}$;

2) infinitesimal Torelli fails for $S_{k}:=C_{1} \times C_{k} / G$;

3) $K_{S_{k}}$ is quasi very ample.

3. Torelli fibres. In this section we want to analyse the above series of examples in the following sense. We know that the infinitesimal Torelli map fails to be injective for reasons of dimension of the eigenspaces, but we want to calculate the dimension of the kernel of the infinitesimal Torelli map.

We start by computing the respective dimensions of $H^{1}\left(S_{k}, \Omega_{S_{k}}^{1}\right), H^{0}\left(S_{k}, \Omega_{S_{k}}^{2}\right)$ and $H^{1}\left(S_{k}, \Omega_{S_{k}}^{1} \otimes \Omega_{S_{k}}^{2}\right)$, and end counting the dimension of the moduli space of the surfaces $S_{k}$.

Let $k$ be any natural number bigger or equal to two and let $P_{1}, \ldots, P_{3 k-2}, P_{3 k-1}$ be pairwise different points in $\mathbb{P}^{1}$. We consider the triple cover $C_{k}$ of $\mathbb{P}^{1}$ ramified along

$$
P_{1}+\ldots+P_{3 k-2}+2 P_{3 k-1} .
$$

Then we define: $S_{k}:=\left(C_{1} \times C_{k}\right) / G$, where $G:=\mathbb{Z} / 3 \mathbb{Z}$. We prove the following

Proposition 3.1. Under the above hypotheses we have: 1) $h^{0}\left(S_{k}, \Omega_{S_{k}}^{2}\right)=6 k-6$, 
2) $h^{1}\left(S_{k}, \Omega_{S_{k}}^{1}\right)=12 k-10$,

3) $h^{1}\left(S_{k}, \Omega_{S_{k}}^{1} \otimes \Omega_{S_{k}}^{2}\right)=h^{1}\left(S_{k}, T_{S_{k}}\right)=3 k+2$.

REMARK 3.2. In particular, it follows from the above proposition that the infinitesimal Torelli theorem does not fail for reasons of dimension of the vector spaces in question, but only because of the imposed symmetry.

Proof. For the dimensions of the eigenspaces of $H^{0}\left(\Omega_{C_{1}}^{1}\right)$, resp. $H^{0}\left(\Omega_{C_{k}}^{1}\right)$, we have the following:
a) $H^{0}\left(C_{1}, \Omega_{C_{1}}^{1}\right)^{0}=\mathbb{C}^{3}$,
b) $H^{0}\left(C_{1}, \Omega_{C_{1}}^{1}\right)^{1}=\mathbb{C}^{2}$,
c) $H^{0}\left(C_{1}, \Omega_{C_{1}}^{1}\right)^{2}=\mathbb{C}^{2}$,
d) $H^{0}\left(C_{k}, \Omega_{C_{k}}^{1}\right)^{0}=0$,
e) $H^{0}\left(C_{k}, \Omega_{C_{k}}^{1}\right)^{1}=H^{0}\left(\mathbb{P}^{1}, \mathcal{O}(2 k-3)\right)=\mathbb{C}^{2 k-2}$,
f) $H^{0}\left(C_{k}, \Omega_{C_{k}}^{1}\right)^{2}=H^{0}\left(\mathbb{P}^{1}, \mathcal{O}(k-2)\right)=\mathbb{C}^{k-1}$.

Using the decomposition of the cohomology groups of $Z_{k}:=C_{1} \times C_{k}$ into eigenspaces corresponding to the characters of $G$ we obtain

$$
\left(H^{0}\left(C_{1}, \Omega_{C_{1}}^{1}\right)^{1} \otimes H^{0}\left(C_{k}, \Omega_{C_{k}}^{1}\right)^{2}\right) \oplus\left(H^{0}\left(C_{1}, \Omega_{C_{1}}^{1}\right)^{2} \otimes H^{0}\left(C_{k}, \Omega_{C_{k}}^{1}\right)^{1}\right)=\mathbb{C}^{6 k-6},
$$

which proves 1).

Moreover,

$$
\begin{gathered}
\left(H^{1}\left(C_{1}, \Omega_{C_{1}}^{1}\right) \otimes H^{0}\left(C_{k}, \mathcal{O}_{C_{k}}\right)\right) \oplus\left(H^{0}\left(C_{1}, \mathcal{O}_{C_{1}}\right) \otimes H^{1}\left(C_{k}, \Omega_{C_{k}}^{1}\right)\right) \oplus \\
\bigoplus_{\chi \in G^{*}}\left(H^{0}\left(C_{1}, \Omega_{C_{1}}^{1}\right)^{\chi} \otimes H^{1}\left(C_{k}, \mathcal{O}_{C_{k}}\right)^{\chi^{*}}\right) \oplus \bigoplus_{\chi \in G^{*}}\left(H^{1}\left(C_{1}, \mathcal{O}_{C_{1}}\right)^{\chi} \otimes H^{0}\left(C_{k}, \Omega_{C_{k}}^{1}\right)^{\chi^{*}}\right)= \\
\mathbb{C}^{2} \oplus\left(H^{0}\left(C_{1}, \Omega_{C_{1}}^{1}\right)^{1} \otimes H^{1}\left(C_{k}, \mathcal{O}_{C_{k}}\right)^{2}\right) \oplus\left(H^{0}\left(C_{1}, \Omega_{C_{1}}^{1}\right)^{2} \otimes H^{1}\left(C_{k}, \mathcal{O}_{C_{k}}\right)^{1}\right) \oplus \\
\oplus\left(H^{1}\left(C_{1}, \mathcal{O}_{C_{1}}\right)^{1} \otimes H^{0}\left(C_{k}, \Omega_{C_{k}}^{1}\right)^{2}\right) \oplus\left(H^{1}\left(C_{1}, \mathcal{O}_{C_{1}}\right)^{2} \otimes H^{0}\left(C_{k}, \Omega_{C_{k}}^{1}\right)^{1}\right)= \\
\mathbb{C}^{2} \oplus \mathbb{C}^{4 k-4} \oplus \mathbb{C}^{2 k-2} \oplus \mathbb{C}^{2 k-2} \oplus \mathbb{C}^{4 k-4}=\mathbb{C}^{12 k-10},
\end{gathered}
$$

and this proves 2 ).

Finally, we see that

$$
\begin{gathered}
H^{1}\left(S_{k}, \Omega_{S_{k}}^{1} \otimes \Omega_{S_{k}}^{2}\right)= \\
\bigoplus_{\chi \in G^{*}}\left(H^{0}\left(C_{1}, \mathcal{O}_{C_{1}}\left(2 K_{C_{1}}\right)\right)^{\chi} \otimes H^{1}\left(C_{k}, \mathcal{O}_{C_{k}}\left(K_{C_{k}}\right)\right)^{\chi^{*}}\right) \oplus \\
\bigoplus_{\chi \in G^{*}}\left(H^{1}\left(C_{1}, \mathcal{O}_{C_{1}}\left(K_{C_{1}}\right)\right)^{\chi} \otimes H^{0}\left(C_{k}, \mathcal{O}_{C_{k}}\left(2 K_{C_{k}}\right)\right)^{\chi^{*}}\right) .
\end{gathered}
$$


Since $H^{1}\left(C_{1}, \mathcal{O}_{C_{1}}\left(K_{C_{1}}\right)\right)$ and $H^{1}\left(C_{k}, \mathcal{O}_{C_{k}}\left(K_{C_{k}}\right)\right)$ are automatically invariant under $G$, we get that

$$
\begin{gathered}
H^{1}\left(S_{k}, \Omega_{S_{k}}^{1} \otimes \Omega_{S_{k}}^{2}\right)=\left(H^{0}\left(C_{1}, \mathcal{O}_{C_{1}}\left(2 K_{C_{1}}\right)\right)^{G} \otimes H^{1}\left(C_{k}, \mathcal{O}_{C_{k}}\left(K_{C_{k}}\right)\right)\right) \oplus \\
\oplus\left(H^{1}\left(C_{1}, \mathcal{O}_{C_{1}}\left(K_{C_{1}}\right)\right) \otimes H^{0}\left(C_{k}, \mathcal{O}_{C_{k}}\left(2 K_{C_{k}}\right)\right)^{G}\right) .
\end{gathered}
$$

Using

$$
H^{0}\left(C_{1}, \mathcal{O}_{C_{1}}\left(2 K_{C_{1}}\right)\right)^{G}=H^{0}\left(C_{1}^{\prime}, \mathcal{O}_{C_{1}^{\prime}}\left(2 K_{C_{1}^{\prime}}\right)\right)=\mathbb{C}^{6}
$$

and

$$
h^{1}\left(C_{1}, \mathcal{O}_{C_{1}}\left(K_{C_{1}}\right)\right)=h^{1}\left(C_{k}, \mathcal{O}_{C_{k}}\left(K_{C_{k}}\right)\right)=1,
$$

we see that we have proven 3) as soon as we have shown that

$$
H^{0}\left(C_{k}, \mathcal{O}_{C_{k}}\left(2 K_{C_{k}}\right)\right)^{G}=\mathbb{C}^{3 k-4} .
$$

This will be done in the following lemma.

First we recall the definition of almost simple cyclic coverings (cf. [Cat89], page 309). Let $Y$ be an algebraic manifold and let $\mathcal{L}=\mathcal{O}_{Y}(F)$ be the invertible sheaf which is the sheaf of sections of a line bundle $\mathbb{L}$ on $Y$. Assume that there are given reduced effective divisors $\Delta_{0}, \Delta_{\infty}$ on $Y$, which are disjoint and it holds $\Delta_{0} \equiv \Delta_{\infty}+n F$.

Definition 3.3. The almost simple cyclic cover associated to $\left(Y, \mathcal{L}, \Delta_{0}, \Delta_{\infty}\right)$ is the subvariety $X \subset \mathbb{P}\left(\mathbb{L} \oplus \mathbb{C}_{Y}\right)$ defined by the equation $z_{1}^{n} \delta_{\infty}=\delta_{0} z_{0}^{n}$, where $\delta_{\infty}$, $\delta_{0}$ are sections defining $\Delta_{\infty}$, resp. $\Delta_{0}$, and $z_{1}, z_{0}$ are respective linear coordinates on the fibres of $\mathbb{L}$, resp. the trivial line bundle $\mathbb{C}_{Y}$.

We have the following :

Lemma 3.4. Let $\pi: X \longrightarrow Y$ be an almost simple cyclic covering of degree $n$ with smooth branch divisors $\Delta_{0}, \Delta_{\infty}$ and set $G:=\mathbb{Z} / n$. Then:

$$
H^{0}\left(X, \mathcal{O}_{X}\left(2 K_{X}\right)\right)^{G}=H^{0}\left(Y, \mathcal{O}_{Y}\left(2 K_{Y}+\Delta_{\infty}+\Delta_{0}\right)\right) .
$$

Proof. $X$ sits in the projective bundle $\mathbb{P}:=\mathbb{P}\left(\mathbb{L} \oplus \mathbb{C}_{Y}\right)=\operatorname{Proj}\left(\mathcal{L}^{-1} \oplus \mathcal{O}_{Y}\right)$ and is linearly equivalent to $n H+p^{*} \Delta_{0}$, where $H$ is the hyperplane divisor $H=\operatorname{div}\left(z_{0}\right)$.

The canonical divisor of $\mathbb{P}$ equals, by the relative Euler sequence, $-2 H+p^{*}\left(K_{Y}-\right.$ $F)$. Thus, by adjunction, $K_{X}$ is the restriction of the divisor

$$
(n-2) H+p^{*}\left(K_{Y}-F+\Delta_{0}\right) .
$$

If we set $E_{i}:=\operatorname{div}\left(z_{i}\right)$, we may write

$$
2 K_{X} \equiv(n-2) E_{0}+(n-2) E_{1}+p^{*}\left(2 K_{Y}+\Delta_{0}+\Delta_{\infty}\right) .
$$

It suffices to show that each invariant section of $H^{0}\left(X, \mathcal{O}_{X}\left(2 K_{X}\right)\right)$ vanishes on $E_{i}$ of multiplicity $n-2$ and after being divided by $\left(z_{0} z_{1}\right)^{n-2}$ yields an invariant section of $H^{0}\left(X, \mathcal{O}_{X}\left(p^{*}\left(2 K_{Y}+\Delta_{\infty}+\Delta_{0}\right)\right)\right)$. 
This follows by a local calculation, since, if we have that $y_{0}=0$ is a local equation of $\Delta_{0}$, we have that $z_{1} / z_{0}:=x_{0}$ is a local equation for the ramification locus $E_{1}$, and we can complete $y_{0}$ to local coordinates $\left(y_{0}, y_{1}, \ldots\right)$ on $Y$.

Note that $\pi$ is locally given by an equation $x_{0}^{n}=y_{0}$, hence $\left(x_{0}, y_{1}, \ldots\right)$ are local coordinates on $X$.

A differential form $f\left(x_{0}, y_{1}, \ldots\right)\left(d x_{0} \wedge d y_{1} \wedge \ldots\right)^{2}$ is $G$ - invariant if and only if $f\left(x_{0}, y_{1}, \ldots\right)=x_{0}^{n-2} \gamma\left(y_{0}, y_{1}, \ldots\right)$.

Hence

$$
\begin{gathered}
f\left(x_{0}, y_{1}, \ldots\right)\left(d x_{0} \wedge d y_{1} \wedge \ldots\right)^{2}=\gamma\left(y_{0}, y_{1}, \ldots\right) x_{0}^{n-2}\left(d x_{0} \wedge d y_{1} \wedge \ldots\right)^{2}= \\
=(n)^{-2} \gamma\left(y_{0}, y_{1}, \ldots\right) x_{0}^{n-2}\left(d y_{0} \wedge d y_{1} \wedge \ldots\right)^{2} \frac{1}{x_{0}^{2 n-2}}=(n)^{-2}\left(d y_{0} \wedge d y_{1} \wedge \ldots\right)^{2} \cdot \frac{\gamma\left(y_{0}, y_{1}, \ldots\right)}{y_{0}} .
\end{gathered}
$$

The same calculation holds for $\Delta_{\infty}$ and we are done.

In our particular case this implies immediately:

COROllary 3.5. Let $k$ be any natural number bigger or equal to two and let $P_{1}, \ldots, P_{3 k-2}, P_{3 k-1}$ be pairwise different points in $\mathbb{P}^{1}$. We consider the triple cover $C_{k}$ of $\mathbb{P}^{1}$ ramified along

$$
P_{1}+\ldots+P_{3 k-2}+2 P_{3 k-1} \text {. }
$$

Then we have

$$
H^{0}\left(C_{k}, \mathcal{O}_{C_{k}}\left(2 K_{C_{k}}\right)\right)^{G} \cong \mathbb{C}^{3 k-4}
$$

Proof. Here $\Delta_{0}=P_{1}+\ldots P_{3 k-2}$ and $\Delta_{\infty}=P_{3 k-1}$ and we get

$$
\begin{gathered}
H^{0}\left(C_{k}, \mathcal{O}_{C_{k}}\left(2 K_{C_{k}}\right)\right)^{G}=H^{0}\left(\mathbb{P}^{1}, \mathcal{O}_{\mathbb{P}^{1}}\left(2 K+\Delta_{0}+\Delta_{\infty}\right)\right) . \\
H^{0}\left(\mathbb{P}^{1}, \mathcal{O}_{\mathbb{P}^{1}}(-4+3 k-1)\right)=H^{0}\left(\mathbb{P}^{1}, \mathcal{O}_{\mathbb{P}^{1}}(3 k-5)\right)=\mathbb{C}^{3 k-4} .
\end{gathered}
$$

We calculate now the dimension of the fibres of the infinitesimal Torelli map.

LEMMA 3.6. Let $k \geq 2$ be a natural number and consider the surfaces $S_{k}$ as above. Then the fibres of the period map $\Phi_{2}$ have (at each point) dimension at least two.

Proof. We know that

$$
\begin{gathered}
\operatorname{dim} \Phi\left(\bigoplus_{\chi \in G^{*}: H^{0}\left(C_{k}, \Omega_{C_{k}}^{1}\right) \chi \neq 0} H^{0}\left(C_{1}, \Omega_{C_{1}}^{1}\right)^{\chi} \otimes H^{0}\left(C_{1}, \Omega_{C_{1}}^{1}\right)^{\chi^{*}}\right) \leq \\
\operatorname{dim}\left(H^{0}\left(C_{1}, \Omega_{C_{1}}^{1}\right)^{1} \otimes H^{0}\left(C_{1}, \Omega_{C_{1}}^{1}\right)^{2}\right)=4,
\end{gathered}
$$

whereas $\operatorname{dim} H^{0}\left(C_{1}, \Omega_{C_{1}}^{1} \otimes \Omega_{C_{1}}^{1}\right)=6$. By the structure of the infinitesimal Torelli map (cf. proof of theorem 2.2) our claim is proven. 
REMARK 3.7. Let $k \geq 2$ be a natural number and let $\mathcal{S}_{k}$ be the family of surfaces we have constructed above, which give an irreducible connected component of the moduli space of surfaces of general type by theorem $\mathrm{C}$ of [Cat00].

Observe that the number of moduli of $S_{k}$ is equal to the sum of the number of moduli of $C_{1}\left(C_{1}\right.$ is a curve of genus three, hence has 6 moduli) and of the number of moduli of the triple cover $C_{k} \rightarrow \mathbb{P}^{1}$ (it is ramified in $3 k-1$ points, whence it has $3 k-1-3=3 k-4$ moduli). From this we conclude that $\operatorname{dim} \mathcal{S}_{k}=3 k+2$.

In particular, since by proposition $3.1,3) h^{1}\left(S_{k}, T_{S_{k}}\right)=3 k+2$, the family $\mathcal{S}_{k}$ yields a generically smooth moduli space, and a smooth base of the Kuranishi family.

We can summarize our results now in the following theorem.

THEOREM 3.8. For any natural number $k \geq 2$ there exists a $3 k+2$ - dimensional family $\mathcal{S}_{k}$ of surfaces such that the following holds:

1) for each $S_{k} \in \mathcal{S}_{k}$ the infinitesimal Torelli map

$$
d \Phi_{2}: H^{1}\left(S_{k}, T_{S_{k}}\right) \longrightarrow \operatorname{Hom}\left(H^{2,0}\left(S_{k}\right), H^{1,1}\left(S_{k}\right)\right)
$$

has a kernel of dimension at least two, i.e., the period map $\Phi_{2}$ has all fibres of dimension strictly positive and $\geq 2$.

2) for a general $S_{k} \in \mathcal{S}_{k}$ the canonical divisor $K_{S_{k}}$ is quasi very ample;

3) $h^{1}\left(S_{k}, T_{S_{k}}\right)=3 k+2$; in particular, $\mathcal{S}_{k}$ yields a generically smooth irreducible connected component of the moduli space.

REMARK 3.9. We observe that, since our examples are irregular surfaces, there is still another period map $\Phi_{1}$ associated to the weight one Hodge structure on $S_{k}$. The differential of this map is given by

$$
d \Phi_{1}: H^{1}\left(S_{k}, T_{S_{k}}\right) \longrightarrow \operatorname{Hom}\left(H^{1,0}\left(S_{k}\right), H^{0,1}\left(S_{k}\right)\right),
$$

and its injectivity is equivalent to the surjectivity of

$$
d \Phi_{1}^{*}: H^{0}\left(S, \Omega_{S}^{1}\right) \otimes H^{1}\left(S, \Omega_{S}^{2}\right) \longrightarrow H^{1}\left(S, \Omega_{S}^{1} \otimes \Omega_{S}^{2}\right) .
$$

Using again the explicit description of $S_{k}$ as in the proof of theorem 2.2, we see that $d \Phi_{1}^{*}$ is surjective if and only if the two maps

$$
\varphi_{i}: H^{0}\left(C_{i}, \Omega_{C_{i}}^{1}\right)^{G} \otimes H^{0}\left(C_{i}, \Omega_{C_{i}}^{1}\right)^{G} \longrightarrow H^{0}\left(C_{i}, \Omega_{C_{i}}^{1} \otimes \Omega_{C_{i}}^{1}\right)^{G}
$$

are surjective. Since $C_{2}^{k}$ is a triple cover of $\mathbb{P}^{1}$ the above map for $i=2$ is obviously not surjective. On the other hand $\varphi_{1}$ is given by the natural map

$$
H^{0}\left(C_{1}^{\prime}, \Omega_{C_{1}^{\prime}}^{1}\right) \otimes H^{0}\left(C_{1}^{\prime}, \Omega_{C_{1}^{\prime}}^{1}\right) \longrightarrow H^{0}\left(C_{1}^{\prime}, \Omega_{C_{1}^{\prime}}^{1} \otimes \Omega_{C_{1}^{\prime}}^{1}\right)
$$

and this map is surjective if and only if $C_{1}^{\prime}$ is not hyperelliptic.

In the case that $C_{1}^{\prime}$ is hyperelliptic, the cokernel of $\varphi_{1}$ has dimension 1 (in general $g-2)$ and is transversal to the cokernel of $\Phi_{2}$.

4. Global double Torelli for surfaces isogenous to a product. Recall that the global Torelli theorem for algebraic curves says that the isomorphism class of a curve $C$ is completely determined by the isomorphism class of the datum of the integral cohomology algebra $H^{*}(C, \mathbb{Z})$ together with the Hodge decomposition

$$
H^{1}(C, \mathbb{Z}) \otimes_{\mathbb{Z}} \mathbb{C}=H^{1,0}(C) \oplus H^{0,1}(C),
$$


where $H^{1,0}(C)=H^{0}\left(C, \Omega_{C}^{1}\right)$ and $H^{0,1}(C)=\overline{H^{1,0}(C)}$.

We shall shortly say that $C$ is determined by its integral Hodge structure.

A fortiori, $C$ is determined by its topological type given together with its Hodge decomposition.

In this section we want to address the question: when does global double Torelli hold for $S=C_{1} \times C_{2} / G$ ? "Double" means that the datum of the respective Hodge structures of weight one and two of $S$, together with the oriented topological type, should determine the isomorphism class of $S$.

To be more precise and explicit, we first observe the following:

Lemma 4.1. Let $G$ be a finite group acting on a Riemann surface $C$ of genus $g \geq 2$. Then the pair consisting of the orbifold group exact sequence and of the Hodge structure of $C$

$$
\left(1 \rightarrow \pi_{1}(C) \rightarrow \pi_{1}^{\text {orb }} \rightarrow G \rightarrow 1, H^{0}\left(\Omega_{C}^{1}\right) \subset H^{1}(C, \mathbb{C})=H^{1}\left(\pi_{1}(C), \mathbb{C}\right)\right)
$$

determines the holomorphic action of $G$ on $C$.

Proof. Inner conjugation on $\pi_{1}(C)$ of a lift of an element $\gamma \in G$ provides an inclusion of $G$ in the mapping class group $G \rightarrow$ Out $\left(\pi_{1}(C)\right)=M a p_{g}$ of $C$, which, together with the natural orientation provided by the half line $\left.H^{0}\left(\Omega_{C}^{1}\right)\right) \wedge \overline{\left.H^{0}\left(\Omega_{C}^{1}\right)\right)} \subset$ $H^{1}(C, \mathbb{R})$, determine the oriented topological type of the action. The Hodge structure determines the complex structure on the curve which makes the $G$-action holomorphic. 口

Assume now that $S$ is a surface isogenous to a product and not of mixed type: by [Cat00], p. 25/26 it follows that $\pi_{1}(S)$ determines the exact sequence

$$
1 \rightarrow \Pi_{g_{1}} \times \Pi_{g_{2}} \rightarrow \pi_{1}(S) \rightarrow G \rightarrow 1
$$

which in turn determines the two orbifold $\pi_{1^{-}}$exact sequences

$$
1 \rightarrow \Pi_{g_{i}} \rightarrow \pi_{1}^{o r b}\left(C_{i}^{\prime}-B, m_{i}^{\prime \prime}\right) \rightarrow G \rightarrow 1 .
$$

Therefore the double Torelli question reduces to the following:

Does the Hodge structure on $S$ determine the Hodge structures on $C_{1}$ and $C_{2}$ ?

REMARK 4.2. Let $\Delta: G \hookrightarrow G \times G$ be the diagonal inclusion.

Then, since $G$ is Abelian, $G=G \times G / \Delta(G)$ acts on the cohomology algebra $H^{*}(S)$ and we get the following decompositions according to the characters (here, $\chi^{*}$ denotes the character of the dual representation, i.e., $\chi^{*}$ is the inverse of $\chi$, and $\left.\chi^{*}=(\chi)^{-1}=\bar{\chi}\right)$ :

$H^{1}(S, \mathbb{Z})$, which is a free $\mathbb{Z}$ - module, contains as a submodule of finite index $H^{1}\left(C_{1} \times C_{2}, \mathbb{Z}\right)^{G}=H^{1}\left(C_{1}, \mathbb{Z}\right)^{G} \oplus H^{1}\left(C_{2}, \mathbb{Z}\right)^{G} ;$

$$
\begin{gathered}
H^{0}\left(S, \Omega_{S}^{1}\right)=H^{0}\left(C_{1}, \Omega_{C_{1}}^{1}\right)^{G} \oplus H^{0}\left(C_{2}, \Omega_{C_{2}}^{1}\right)^{G} ; \\
H^{1}\left(C_{i}, \mathbb{C}\right)=\bigoplus_{\chi \in G^{*}} H^{1}\left(C_{i}, \mathbb{C}\right)^{\chi}=\bigoplus_{\chi \in G^{*}}\left(H^{0}\left(C_{i}, \Omega_{C_{i}}^{1}\right)^{\chi} \oplus \overline{H^{0}\left(C_{i}, \Omega_{C_{i}}^{1}\right)^{\chi^{*}}}\right)= \\
=\bigoplus_{\chi \in G^{*}}\left(H^{0}\left(C_{i}, \Omega_{C_{i}}^{1}\right)^{\chi} \oplus H^{1}\left(C_{i}, \mathcal{O}_{C_{i}}\right)^{\chi}\right) ;
\end{gathered}
$$




$$
H^{0}\left(S, \Omega_{S}^{2}\right)=\bigoplus_{\chi \in G^{*}}\left(H^{0}\left(C_{1}, \Omega_{C_{1}}^{1}\right)^{\chi} \otimes H^{0}\left(C_{2}, \Omega_{C_{2}}^{1}\right)^{\chi^{*}}\right)
$$

We will use in the sequel the following quite elementary but very useful result.

Lemma 4.3. Let $0 \neq U^{\prime} \subset U$ and $0 \neq V^{\prime} \subset V$ be complex vector spaces and set $L:=U^{\prime} \otimes V^{\prime} \subset U \otimes V$. Then the subspace $L$ of $U \otimes V$ determines $U^{\prime}$ and $V^{\prime}$.

Proof. Let $\dashv$ denote the contraction operator $\dashv:(U \otimes V) \times V^{\vee} \rightarrow U$. Then $L \dashv V^{\vee}=U^{\prime}$. Similarly we get $L \dashv U^{\vee}=V^{\prime}$.

We will see that global double Torelli holds for a huge class of surfaces isogenous to a product of curves, but nevertheless there are also lots of potential counterexamples. Our first result is the following:

THEOREM 4.4. Let $G$ be a finite abelian group acting on two curves $C_{1}, C_{2}$ of respective genera $g_{1}, g_{2} \geq 2$ and acting freely by the product action on $Z:=C_{1} \times$ $C_{2}$. Double global Torelli holds for $S:=C_{1} \times C_{2} / G$., i.e., the Hodge structure of $S$ determines the Hodge structures of $C_{1}$ and $C_{2}$, under the following hypothesis:

A) $C_{i} / G$, for $i=1,2$, has either genus $\geq 2$ or it has genus 1 but there is no nontrivial subgroup $H$ of $G$ such that $C / H$ has genus 1 .

Proof. Since every automorphism leaves the fundamental class of $C_{i}$ invariant, we have $H^{2}\left(C_{i}, \mathbb{C}\right)=H^{2}\left(C_{i}, \mathbb{C}\right)^{G}=H^{1}\left(C_{i}, \Omega_{C_{i}}^{1}\right)^{G}=H^{1}\left(C_{i}, \Omega_{C_{i}}^{1}\right)$.

Therefore the proof reduces to the following problem:

We know that $H^{1}\left(C_{i}, \mathbb{C}\right)=\bigoplus_{\chi \in G^{*}} H^{1}\left(C_{i}, \mathbb{C}\right)^{\chi}$ and we have to recover the decomposition

$$
H^{1}\left(C_{i}, \mathbb{C}\right)^{\chi}=H^{0}\left(C_{i}, \Omega_{C_{i}}^{1}\right)^{\chi} \oplus \overline{H^{0}\left(C_{i}, \Omega_{C_{i}}^{1}\right)^{\chi^{*}}}
$$

for each $\chi \in G^{*}$.

Obviously, if for $\chi \in G^{*}$ one of the two above summands is zero, we are done for this $\chi$, once we know which of the two summands is equal to zero.

0 .

Therefore, let $\chi$ be a character such that $H^{0}\left(C_{i}, \Omega_{C_{i}}^{1}\right)^{\chi} \neq 0$ and $H^{0}\left(C_{i}, \Omega_{C_{i}}^{1}\right)^{\chi^{*}} \neq$ Using

$$
H^{0}\left(S, \Omega_{S}^{2}\right)=\bigoplus_{\chi}\left(H^{0}\left(C_{1}, \Omega_{C_{1}}^{1}\right)^{\chi} \otimes H^{0}\left(C_{2}, \Omega_{C_{2}}^{1}\right)^{\chi^{*}}\right)
$$

and

$$
H^{1}\left(C_{i}, \mathbb{C}\right)^{\chi}=H^{0}\left(C_{i}, \Omega_{C_{i}}^{1}\right)^{\chi} \oplus \overline{H^{0}\left(C_{i}, \Omega_{C_{i}}^{1}\right)^{\chi^{*}}}
$$

we see that the contraction

$$
H^{0}\left(S, \Omega_{S}^{2}\right) \dashv H^{1}\left(C_{2}, \mathbb{C}\right)^{\chi} \longrightarrow H^{0}\left(C_{1}, \Omega_{C_{1}}^{1}\right)^{\chi}
$$

is surjective, if $H^{0}\left(C_{2}, \Omega_{C_{2}}^{1}\right)^{\chi *} \neq 0$. Using that $g(C / G) \geq 1$ implies $H^{0}\left(C, \Omega_{C}^{1}\right)^{\chi} \neq 0$ (cf. following proposition) we are done.

Proposition 4.5. Let $C$ be a smooth algebraic curve of genus at least 2 and suppose we have an effective action of $G:=\mathbb{Z} / d$ on $C$.

1) Let $\chi \in G^{*}$ : if $g(C / G) \geq 2$, or $g(C / G)=1$ and $\chi$ is a primitive character (mod d), then $H^{0}\left(C, \Omega_{C}^{1}\right)^{\chi} \neq 0$. 
2) Let $G_{\chi}$ be the subgroup which is the kernel of a character $\chi$ and suppose that $g\left(C / G_{\chi}\right) \geq 2$ : if $g(C / G)=1$, then $H^{0}\left(C, \Omega_{C}^{1}\right)^{\chi} \neq 0$. If $g(C / G)=0$ then either $H^{0}\left(C, \Omega_{C}^{1}\right)^{\chi} \neq 0$ or $H^{0}\left(C, \Omega_{C}^{1}\right)^{\chi *} \neq 0$.

Clearly, if $\chi \neq 0$, and $g\left(C / G_{\chi}\right)=0$, then $H^{0}\left(C, \Omega_{C}^{1}\right)^{\chi}=H^{0}\left(C, \Omega_{C}^{1}\right)^{\chi^{*}}=0$. If instead $g\left(C / G_{\chi}\right)=1$ and $\left|G / G_{\chi}\right| \geq 3$, either $H^{0}\left(C, \Omega_{C}^{1}\right)^{\chi}=0$ or $H^{0}\left(C, \Omega_{C}^{1}\right)^{\chi *}=0$.

3) If $g(C / G)=0$, then, assuming that the number of branch points is at least 4 , the cardinality of the set $\left\{\chi \in G^{*}: H^{0}\left(C, \Omega_{C}^{1}\right)^{\chi}=0\right\}$ is strictly smaller than $\frac{d-1}{2}$.

Proof. 1) Let $P_{1}, \ldots, P_{r} \in Y$ be the branch points of the map $C \rightarrow C / G=: Y$.

After we fix a generator for the group $G$, thus also for the group of characters $G^{*}$, each $P_{i}$ determines an isotropy subgroup $H_{i} \cong \frac{d}{d_{i}^{\prime}} \mathbb{Z} / d \mathbb{Z} \subset \mathbb{Z} / d \mathbb{Z}$ and a character of the representation of $H_{i}$ on the tangent space $T_{C, Q_{i}}, Q_{i}$ being any point lying over $P_{i}$.

As we shall see later, representing this character by an integer $a_{i}\left(\bmod d_{i}^{\prime}\right)$, with $\left(a_{i}, d_{i}^{\prime}\right)=1$, we obtain $m_{i}^{\prime}$ by the equation $a_{i} m_{i}^{\prime} \equiv 1\left(\bmod d^{\prime}\right)$. We then obtain natural numbers $1 \leq m_{j} \leq d-1$ setting $m_{j}:=m_{j}^{\prime} \frac{d}{d_{j}^{\prime}}$ and we have

$$
d L \equiv \sum m_{j} P_{j}=: \hat{B}
$$

Let $\sigma_{j}$ be the unique section of $\mathcal{O}_{Y}\left(P_{j}\right)$ vanishing on $P_{j}$ : then the equation $z^{d}=\prod \sigma_{j}^{m_{j}}$ in $\mathbb{L}$ defines a singular covering $p: X \rightarrow Y$ (such that $C$ is the normalization of $X$ ). We have

$$
p_{*} \omega_{X}=\omega_{Y} \oplus \omega_{Y}(L) \oplus \ldots \oplus \omega_{Y}\left(L^{d-1}\right) .
$$

A local generator of $\omega_{Y}\left(L^{i}\right)$ is given by $d x \cdot z^{-i}$ and at $P_{j}$ we have (setting $m:=m_{j}$, $x=\sigma_{j}$ ) the local equation $z^{d}=x^{m}$ for $X \subset \mathbb{L}$.

We will investigate now when is $\varphi(x) d x \cdot z^{-i}$ regular on $C$.

Let $r$ be the greatest common divisor of $d$ and $m$, and write $m=r m^{\prime}, d=r d^{\prime}$. Then the equation $z^{d}=x^{m}$ decomposes in

$$
\prod_{\epsilon^{r}=1}\left(z^{d^{\prime}}-\epsilon x^{m^{\prime}}\right)=0 .
$$

We choose a point on the normalization $C$ of $X$ and let $t$ be a local coordinate of $C$ around this point. Then the cyclic group $H_{j} \cong\left\{\zeta \mid \zeta^{d^{\prime}}=1\right\}$ acts locally by sending

$$
z \rightarrow \zeta z, t \rightarrow \zeta^{a} t
$$

Moreover, the equations

$$
\begin{gathered}
z=t^{m^{\prime}} \epsilon^{\frac{1}{d^{\prime}}}, \\
x=t^{d^{\prime}}
\end{gathered}
$$

give a parametrisation of the branch of $C$ over $P_{j}$. Then $\varphi(x) d x \cdot z^{-i}$ (on $Y$ ) pulls back to

$$
\left.\varphi\left(t^{d^{\prime}}\right) d\left(t^{d^{\prime}}\right)\left(t^{m^{\prime}} \epsilon^{\frac{1}{d^{\prime}}}\right)^{-i}=d^{\prime} t^{d^{\prime}-1} \varphi\left(t^{d^{\prime}}\right) d t \cdot t^{-i m^{\prime}} \cdot \epsilon^{\frac{-i}{d^{\prime}}}\right)
$$

This is regular iff $\varphi(x) x \cdot x^{-\frac{i m^{\prime}+1}{d^{\prime}}}$ has order at least 0 , i.e. iff $\operatorname{ord} \varphi+1 \geq \frac{i m^{\prime}+1}{d^{\prime}}$, or, equivalently, $\lceil y\rceil:=-[-y]$ denoting the round up of a real number $y$, iff $\operatorname{ord} \varphi \geq$ 
$\left\lceil\frac{i m^{\prime}+1}{d^{\prime}}\right\rceil-1=\left\lceil\frac{i m+r}{d}\right\rceil-1$.

Therefore we have been able to compute the eigenspace for the $i-t h$ character as:

$$
H^{0}\left(C ; \Omega_{C}^{1}\right)^{i}=H^{0}\left(Y, \mathcal{L}_{i}\right)
$$

where

$$
\mathcal{L}_{i}=\omega_{Y}\left(i L-\sum_{j}\left(\left\lceil\frac{i m_{j}+r_{j}}{d}\right\rceil-1\right)\right)
$$

is a line bundle of degree

$$
2 g(Y)-2+\sum_{j}\left(\frac{i m_{j}}{d}-\left(\left\lceil\frac{i m_{j}+r_{j}}{d}\right\rceil-1\right)\right) .
$$

We remark that

$\frac{i m_{j}}{d}-\left(\left\lceil\frac{i m_{j}+r_{j}}{d}\right\rceil-1\right)=\frac{i m_{j}^{\prime}}{d^{\prime}}-\left(\left\lceil\frac{i m_{j}^{\prime}+1}{d^{\prime}}\right\rceil-1\right)=\frac{i m_{j}^{\prime}}{d^{\prime}}+1-\left(\left[\frac{i m_{j}^{\prime}}{d^{\prime}}\right]+1\right)=\left\{\frac{i m_{j}^{\prime}}{d^{\prime}}\right\}$.

I.e., we obtain that the summands provide exactly the fractionary part $\left\{\frac{i m_{j}}{d}\right\}$ of $\frac{i m_{j}}{d}$, in other words, the remainder class of $i m_{j}(\bmod d)$, divided by $d$.

Therefore if $g(Y) \geq 2$ we see that $\operatorname{deg} \mathcal{L}_{i} \geq 2 g(Y)-2 \geq 2$, whence we get $h^{0}\left(C, \Omega_{C}^{1}\right)^{i} \geq g(Y)-1 \neq 0$.

If instead $g(Y)=1$ and $\chi$ is a primitive character, then the G.C.D. between $i$ and $d$ is 1 , whence $\left\{\frac{i m_{j}}{d}\right\}>0$ for each $m_{j} \neq 0$ : we conclude since, the genus of $C$ being $\geq 2$, there is at least one $m_{j} \neq 0$.

2) If $\chi$ is a non primitive character, we simply observe that $\chi$ yields a primitive character $\chi^{\prime}$ of $G / G_{\chi}$, and $H^{0}\left(C, \Omega_{C}^{1}\right)^{\chi}$ is the pull back of $H^{0}\left(C / G_{\chi}, \Omega_{C}^{1}\right)^{\chi^{\prime}}$, so that the first assertion is a direct consequence of 1$)$.

Assume now that $g\left(C / G_{\chi}\right) \geq 2$ and $g(C / G)=0$. We apply the basic estimate we used in the proof of 1$)$ : i.e., we have a primitive character and then $H^{0}\left(C, \Omega_{C}^{1}\right)^{\chi} \neq 0$ if $\sum_{j}\left\{\frac{i m_{j}}{d}\right\} \geq 2$.

Without loss of generality, since we have a primitive character, we may assume $i=1$. $H^{0}\left(C, \Omega_{C}^{1}\right)^{\chi} \neq 0$ unless $\sum_{j} m_{j}=d$. But our assertion holds since the dual character corresponds to $d-1$, and $H^{0}\left(C, \Omega_{C}^{1}\right)^{\chi *} \neq 0$ unless $\sum_{j}\left(d-m_{j}\right)=d$. This is a contradiction, since then $2 d=\sum_{j}\left(d-m_{j}+m_{j}\right)=d\left(\sum_{j} 1\right)$, there are exactly $r=2$ branch points, whence $g\left(C / G_{\chi}\right)=0$.

3) Assume finally $g(Y)=0$, i.e. $Y=\mathbb{P}^{1}$. Obviously $H^{0}\left(C, \Omega_{C}^{1}\right)^{0}=H^{0}\left(Y, \Omega_{Y}^{1}\right)=$ 0 . For $i>0$ we have, by our previous calculation:

$$
H^{0}\left(C, \Omega_{C}^{1}\right)^{i} \neq 0
$$

if and only if

$$
\sum_{j}\left\{\frac{i m_{j}}{d}\right\} \geq 2
$$

Then the assertion follows from the following lemma. $\mathrm{a}$ 
LEMMA 4.6. Let $r, d, m_{1}, \ldots m_{r}$ be natural numbers such that $r \geq 4$ and $m_{1}+$ $\ldots m_{r}=d$. We define for each $1 \leq i \leq d-1$ a natural number $\lambda(i)$ by the equality

$$
\overline{i m_{1}}+\ldots \overline{i m_{r}}=\lambda(i) d
$$

where $0 \leq \bar{z} \leq d-1$ denotes the remainder modulo $d$ of a natural number $z$. Then

$$
\sharp\{i: \lambda(i)=1\}<\frac{d-1}{2} \text {. }
$$

Proof. Assume that $r>4$. Then we set $M_{r-1}:=m_{r-1}+m_{r}$ and it follows

$$
\lambda^{r}(i) d=\overline{i m_{1}}+\ldots \overline{i m_{r}} \geq \overline{i m_{1}}+\ldots \overline{i M_{r-1}}=\lambda^{r-1}(i) d .
$$

Therefore without restriction we can assume $r=4$. For $d=4$ we have $m_{1}=m_{2}=$ $m_{3}=m_{4}=1$ and the claim is obvious. Let now $d+1>4$ and $d+1=m_{1}+\ldots+m_{4}^{\prime}$. Without restriction we can assume that $m_{4}^{\prime}>1$, hence we write $m_{4}^{\prime}=m_{4}+1$. Then $m_{1}+\ldots+m_{4}=d$ and by induction we get $\sum_{j}{\overline{i m_{j}}}^{d}=\lambda^{\prime}(i) d$ and $\sharp\left\{i: \lambda^{\prime}(i)=1\right\}<$ $\frac{d-1}{2}$.

We write for $1 \leq j \leq 3$ :

$$
i m_{j}=a_{j} d+{\overline{i m_{j}}}^{d}=b_{j}(d+1)+{\overline{i m_{j}}}^{d+1}
$$

and

$$
i\left(m_{4}+1\right)=\gamma d+{\overline{i m_{4}}}^{d}+i=c(d+1)+{\overline{i\left(m_{4}+1\right)}}^{d+1} .
$$

Then

$$
{\overline{i\left(m_{4}+1\right)}}^{d+1}={\overline{i m_{4}}}^{d}+(\gamma-c) d+i-c,
$$

whence

$$
\begin{gathered}
\lambda(i)(d+1)={\overline{i m_{1}}}^{d+1}+\ldots+{\overline{i\left(m_{4}+1\right)}}^{d+1}= \\
={\overline{i m_{1}}}^{d}+\ldots+{\overline{i m_{4}}}^{d}+\left(a_{1}-b_{1}\right) d+\ldots+\left(a_{3}-b_{3}\right) d+(\gamma-c) d+i-b_{1}-b_{2}-b_{3}-c .
\end{gathered}
$$

We remark that

$$
i d=\left(a_{1}+a_{2}+a_{3}+\gamma\right) d+\lambda^{\prime}(i) d .
$$

Therefore $\lambda^{\prime}(i)=1$ implies that $a_{1}+a_{2}+a_{3}+\gamma=i-1$. Analogously $\lambda(i)=1$ implies that $b_{1}+b_{2}+b_{3}+c=i-1$.

Assume that $\lambda(i)=1$ : then

$$
\lambda^{\prime}(i) d+\left(a_{1}-b_{1}\right) d+\ldots+(\gamma-c) d+1=d+1 .
$$

Since $a_{1}-b_{1}, \ldots, \gamma-c \geq 0$ we see that the above equality implies that $\lambda^{\prime}(i)=1$. Therefore $\sharp\{i \leq d-1: \lambda(i)=1\}<\frac{d-1}{2}$ and we are done if we show that $\lambda(d) \neq 1$.

Since

$$
\lambda(d)(d+1)={\overline{d m_{1}}}^{d+1}+\ldots+{\overline{d\left(m_{4}+1\right)}}^{d+1}
$$

if $\lambda(d)=1$ we get

$$
\begin{aligned}
& 2(d+1)=m_{1}+\ldots+\left(m_{4}+1\right)+{\overline{d m_{1}}}^{d+1}+\ldots+{\overline{d\left(m_{4}+1\right)}}^{d+1}= \\
& =\left(m_{1}+{\overline{d m_{1}}}^{d+1}\right)+\ldots+\left(\left(m_{4}+1\right)+{\overline{d\left(m_{4}+1\right)}}^{d+1}\right)=4(d+1),
\end{aligned}
$$

which is absurd. 
5. Another series of examples. In the following we will construct a series of examples of surfaces isogenous to a higher product, for which we are not able to prove global double Torelli using theorem 4.4 or proposition 4.5. It turns out nevertheless that for these surfaces the local period map is injective.

It seems therefore an interesting problem to decide whether global double Torelli holds for this class of surfaces (or for similar classes).

We consider for any natural number $d \geq 3$ the group $G=G_{d}=\mathbb{Z} / d \oplus \mathbb{Z} / d$. Let $\pi: C_{1} \longrightarrow C_{1} / G=\mathbb{P}^{1}$ be the covering branched in $P, P^{\prime}$ (with local monodromy given by $(1,0)$, respectively $(-1,0))$ and in $P_{1}, \ldots P_{d}$ (with $(0,1)$ as local monodromy).

$C_{1}$ is then the curve in the weighted projective plane $\mathbb{P}(1,1, d)$ with coordinates $\left(x_{0}, x_{1}, z\right)$ defined by the equation (homogeneous of degree $\left.d^{2}\right)$

$$
z^{d}=\prod_{j=1}^{d}\left(x_{1}^{d}-\alpha_{j} x_{0}^{d}\right) .
$$

$\pi$ is given by $\pi\left(x_{0}, x_{1}, z\right):=\left(x_{0}^{d}, x_{1}^{d}\right)$. We set for convenience $x:=x_{1} / x_{0}, u:=x^{d}$, $u_{0}:=x_{0}^{d}, u_{1}:=x_{1}^{d}$.

Then the canonical sheaf of $C_{1}$ is $\mathcal{O}_{C_{1}}\left(d^{2}-d-2\right)$ and it is easy to see that, in affine coordinates, the space of holomorphic 1-forms can be written as follows, where the $F_{j}(x)$ 's are polynomials of degree $\leq j$ :

$$
H^{0}\left(C_{1}, \Omega_{C_{1}}^{1}\right)=\left\{F_{d-2}(x) \frac{d x}{z}+F_{2 d-2}(x) \frac{d x}{z^{2}}+\ldots+F_{d(d-1)-2}(x) \frac{d x}{z^{d-1}}\right\} .
$$

Accordingly, the canonical map is given by $\Phi\left(x_{0}, x_{1}, z\right)=\left(z^{i} x_{0}^{h} x_{1}^{r}\right)$, where $0 \leq i \leq$ $d-2, h+r+d i=\left(d^{2}-d-2\right)$.

We can now write the cohomology table of $C_{1}$, where we write in the place $(a, b)$ the dimension of the eigenspace of $H^{0}\left(C_{1}, \Omega_{C_{1}}^{1}\right)$ belonging to the character $(a, b)$, i.e. $\operatorname{dim} H^{0}\left(C_{1}, \Omega_{C_{1}}^{1}\right)^{(a, b)}$, noting that $\frac{d x \cdot x^{i}}{z^{j}}$ belongs to the character $(-j, \overline{i+1})$.

\begin{tabular}{|l|c|c|c|c|c|c|}
\hline & $a=0$ & $a=1$ & $a=2$ & $a=3$ & $\ldots$ & $a=d-1$ \\
\hline \hline$b=0$ & 0 & 0 & 1 & 2 & $\ldots$ & $d-2$ \\
\hline$b=1$ & 0 & 1 & 2 & 3 & $\ldots$ & $d-1$ \\
\hline$\ldots$ & $\ldots$ & $\ldots$ & $\ldots$ & $\ldots$ & $\ldots$ & $\ldots$ \\
\hline$b=d-2$ & 0 & 1 & 2 & 3 & $\ldots$ & $d-1$ \\
\hline$b=d-1$ & 0 & 1 & 2 & 3 & $\ldots$ & $d-1$ \\
\hline
\end{tabular}

We take now the covering $\varphi: C_{2} \longrightarrow C_{2} / G=\mathbb{P}^{1}$ branched in $P, P^{\prime}$ (with local monodromies $(1,0)$ and $(-1,0)$ ) and in $Q, Q^{\prime}$ (with local monodromies $(0,1),(0,-1)$ ).

We may assume without loss of generality that the points $P, P^{\prime}$ are the respective points $v=0, v=\infty$, whereas the points $Q, Q^{\prime}$ are the respective points $v=1, v=-\lambda$.

We see easily that $C_{2} \subset \mathbb{P}^{1} \times \mathbb{P}^{1}$ is given by the equation

$$
w_{1}^{d}\left(y_{1}^{d}+\lambda y_{0}^{d}\right)=w_{0}^{d}\left(y_{1}^{d}-y_{0}^{d}\right),
$$

$\varphi\left(\left(y_{0}, y_{1}\right)\left(w_{0}, w_{1}\right)\right):=\left(y_{0}^{d}, y_{1}^{d}\right)$ and in affine coordinates $C_{2}$ is the fibre product of two cyclic coverings:

$$
y^{d}=v,
$$




$$
w^{d}=\frac{v-1}{v+1} .
$$

We see immediately that

$$
H^{0}\left(C_{2}, \Omega_{C_{2}}^{1}\right)=\left\{F_{\leq d-2, \leq d-2}(w, y) \frac{d y}{w^{d-1}\left(y^{d}+1\right)}\right\}=\left\{F_{\leq d-2, \leq d-2}(w, y) \frac{w d y}{v-1}\right\} .
$$

Therefore we have the following cohomology table:

\begin{tabular}{|l|c|c|c|c|c|c|}
\hline & $a=0$ & $a=1$ & $a=2$ & $a=3$ & $\ldots$ & $a=d-1$ \\
\hline \hline$b=0$ & 0 & 0 & 0 & 0 & $\ldots$ & 0 \\
\hline$b=1$ & 0 & 1 & 1 & 1 & $\ldots$ & 1 \\
\hline$\ldots$ & $\ldots$ & $\ldots$ & $\ldots$ & $\ldots$ & $\ldots$ & $\ldots$ \\
\hline$b=d-2$ & 0 & 1 & 1 & 1 & $\ldots$ & 1 \\
\hline$b=d-1$ & 0 & 1 & 1 & 1 & $\ldots$ & 1 \\
\hline
\end{tabular}

In order to have $G$ operating freely on the product $C_{1} \times C_{2}$ we have to twist the action of $G$ on $C_{2}$. We assume for simplicity that $d$ is a prime number. Let now $r \neq 0$ and $\neq 1$. Then twisting the action of $C_{2}$ by the automorphism of $\mathbb{Z} / d \mathbb{Z} \oplus \mathbb{Z} / d \mathbb{Z}$ given by

$$
(1,0) \mapsto(1,1), \quad(0,1) \mapsto(r, 1),
$$

we see that the stabilizers of the twisted action on $C_{2}$ are now $\langle(1,1)\rangle$ and $<(r, 1)>$, whence $G$ acts now freely on $C_{1} \times C_{2}$ and the cohomology table of $C_{2}$ becomes now:

\begin{tabular}{|l|c|c|c|c|c|c|}
\hline & $a=0$ & $a=1$ & $a=2$ & $a=3$ & $\ldots$ & $a=d-1$ \\
\hline \hline$b=0$ & 0 & 1 & 1 & 1 & $\ldots$ & 1 \\
\hline$b=1$ & 1 & 0 & $*$ & $*$ & $\ldots$ & $*$ \\
\hline$\ldots$ & $\ldots$ & $\ldots$ & $\ldots$ & $\ldots$ & $\ldots$ & $\ldots$ \\
\hline$b=d-2$ & 1 & $*$ & $*$ & $*$ & $\ldots$ & $*$ \\
\hline$b=d-1$ & 1 & $*$ & $*$ & $*$ & $\ldots$ & 0 \\
\hline
\end{tabular}

I.e., the diagonal is zero and the remaining zeroes are at the places $(n r, n)$, where $0 \leq n \leq d-1$. In particular we see that e.g.

$$
H^{0}\left(C_{1}, \Omega_{C_{1}}^{1}\right)^{(1,1)} \neq 0 \neq H^{0}\left(C_{1}, \Omega_{C_{1}}^{1}\right)^{(d-1, d-1)}
$$

whereas

$$
H^{0}\left(C_{2}, \Omega_{C_{2}}^{1}\right)^{(1,1)}=0=H^{0}\left(C_{2}, \Omega_{C_{2}}^{1}\right)^{(d-1, d-1)} .
$$

Therefore we cannot reconstruct $H^{0}\left(C_{1}, \Omega_{C_{1}}^{1}\right)^{(1,1)}$ as well as $H^{0}\left(C_{1}, \Omega_{C_{1}}^{1}\right)^{(d-1, d-1)}$ from the Hodge structure of $S$.

By theorem 2.2 we can now easily verify that the infinitesimal Torelli map is injective. In fact, $H^{0}\left(C_{i}, \mathcal{O}_{C_{i}}\left(2 K_{C_{i}}\right)\right)^{G}=H^{0}\left(\mathbb{P}^{1}, \mathcal{O}_{\mathbb{P}^{1}}\left(-4+r_{i}\right)\right.$, where $r_{i}$ is the number of branch points of $C_{i} \rightarrow \mathbb{P}^{1}$.

For $i=2$ we get a space of dimension 1 , therefore it suffices to observe that there is a non zero summand in

$$
\bigoplus_{\chi \in G^{*}: H^{0}\left(C_{1}, \Omega_{C_{1}}^{1}\right) \chi \neq 0} H^{0}\left(C_{2}, \Omega_{C_{2}}^{1}\right)^{\chi} \otimes H^{0}\left(C_{2}, \Omega_{C_{2}}^{1}\right)^{\chi^{*}} .
$$


For $i=1$ we must obtain all monomials of degree $d-2$ in $\left(u_{0}, u_{1}\right)$, and for this purpose it suffices, since the pairing is non degenerate, to find a character space $H^{0}\left(C_{1}, \Omega_{C_{1}}^{1}\right)^{\chi}$ of dimension $d-1$ such that the character space $H^{0}\left(C_{1}, \Omega_{C_{1}}^{1}\right)^{\chi *}$ is non zero, and such that likewise $H^{0}\left(C_{2}, \Omega_{C_{2}}^{1}\right)^{\chi} \neq 0$.

We omit here to prove the following

Proposition 5.1. The canonical system of $S$ has base points, and the canonical map is birational onto its image.

Acknowledgement. We would like to thank Gerard van der Geer for providing numerical evidence for Lemma 4.6 when we conjectured it.

\section{REFERENCES}

[Andr58] A. Andreotti, On a theorem of Torelli, Am. J. Math., 80 (1958), pp. 801-828.

[BPV] Barth, W., Peters, C., VAn de Ven, A., Compact complex surfaces, Ergebnisse der Mathematik und ihrer Grenzgebiete (3), Springer-Verlag, Berlin, (1984).

[Bea] A. Beauville, Surfaces algébriques complexes, Asterisque 54 Soc. Math. France (1978).

[Cam32] L. CAmpedelli, Sopra alcuni piani doppi notevoli con curve di diramazione del decimo ordine, Atti Acad. Naz. Lincei, 15 (1932), pp. 536-542.

[Cat84] F. CATANeSE, Infinitesimal Torelli theorems and counterexamples to Torelli problems, Topics in transcendental algebraic geometry (Princeton, N.J., 1981/1982), Ann. of Math. Stud., 106, Princeton Univ. Press, Princeton, NJ, (1984), pp. $143-156$.

[Cat89] F. CATANESE, Everywhere nonreduced moduli spaces, Invent. Math. 98:2 (1989), pp. $293-310$.

[Cat00] F. CATANESE, Fibred surfaces, varieties isogenous to a product and related moduli spaces, Am. J. of Math., 122 (2000), pp. 1-44.

[Cat03] F. CATAnese, Moduli spaces of surfaces and real structures, Ann. of Math., 158 (2003), pp. 539-554.

[Cox87] D. A. Cox, Generic Torelli and infinitesimal variation of Hodge structure, Algebraic geometry, Bowdoin, 1985 (Brunswick, Maine, 1985), Proc. Sympos. Pure Math., 46, Part 2, Amer. Math. Soc., Providence, RI, (1987) pp. 235-246.

[Don84] R. DonAGI, Generic Torelli and variational Schottky, Topics in transcendental algebraic geometry (Princeton, N.J., 1981/1982), Ann. of Math. Stud., 106, Princeton Univ. Press, Princeton, NJ, (1984) pp. 239-258.

[Flen86] H. Flenner, The infinitesimal Torelli problem for zero sets of sections of vector bundles, Math. Z., 193:2 (1986), pp. 307-322.

[God35] L Godeaux, Les involutions cycliques appartenant á une surface algébrique, Actual. Sci. Ind., 270, Hermann, Paris, (1935).

[Gren84] M. L. GREen, Koszul cohomology and the geometry of projective varieties. II, J. Differential Geom., 20:1 (1984), pp. 279-289.

[Gren85] M. L. GREEN, The period map for hypersurface sections of high degree of an arbitrary variety, Compositio Math., 55:2 (1985), pp. 135-156.

[Grif68] P. Griffiths, Periods of integrals on algebraic manifolds I,II, Amer. J. Math., 90 (1968), pp. 568-626, 805-865.

[Grif70] P. Griffiths, Periods of integrals on algebraic manifolds III. Publ. Math. I.H.E.S., 38 (1970), pp. 125-180.

[Grif84] P. GRIfFiths, (ed) Topics in transcendental algebraic geometry, Annals of Math. Studies, 106, Princeton Univ. Press, (1984), pp. 316.

[Grif-Schmid] P. GRIfFiths, W. Schmid, Recent developments in Hodge theory: a discussion of techniques and results, in ' Discrete subgroups of Lie groups and applicatons to moduli' (Internat. Colloq., Bombay, 1973), Oxford Univ. Press, Bombay, (1975), pp. 31-127.

[K-M] K. Kodaina, J. Morrow, Complex Manifolds, Holt - Rinehart - Winston, New York (1971). 
[Kur65] M. Kuranishi, New proof for the existence of locally complete families of complex structures, In: Proc. Conf. Complex Analysis, Minneapolis, Springer, New York (1965), pp. 142-154.

[L-P-W77] D. Lieberman, C. Peters, R. Wilsker, A theorem of local-Torelli type, Math. Ann., 231:1 (1977/78), pp. 39-45.

[Nara96] J. C. Naranjo, The positive-dimensional fibres of the Prym map, Pacific J. Math., 172:1 (1996), pp. 223-226.

[Pa91] R. PARDINI, Abelian covers of algebraic varieties, J. Reine Angew. Mathematik, 417 (1991), pp. 191-213.

[Tor13] R. TorelLi, Sulle varieta' di Jacobi, I, II, Rendiconti R. Accad. dei Lincei, 22-2 (1913), pp. 98-103, 437-441.

[Usui81] S. UsuI, Effect of automorphisms on variation of Hodge structures, J. Math. Kyoto Univ., 21:4 (1981), pp. 645-672.

[Weil-57] A. WeIL, Zum Beweis des Torellischen Satzes, Nachr. Akad. Wiss. Göttingen, Math.-Phys. Kl., IIa 1957, pp. 32-53 (1957).

[Weil-CPII] A. WeIL, Final report on contract AF 18 (603)-57, in : Scientific works. Collected papers. Vol. II (1951-1964), Springer-Verlag, New York-Heidelberg, (1979) xii +561 . 